\title{
Regions of the Cf-9B Disease Resistance Protein Able to Cause Spontaneous Necrosis in Nicotiana benthamiana Lie Within the Region Controlling Pathogen Recognition in Tomato
}

\author{
Apratim Chakrabarti, ${ }^{1}$ Stephen N. Panter, ${ }^{1}$ Kate Harrison, ${ }^{2}$ Jonathan D. G. Jones, ${ }^{2}$ and David A. Jones ${ }^{1}$ \\ ${ }^{1}$ Research School of Biology, The Australian National University, Canberra ACT 0200, Australia; ${ }^{2}$ Sainsbury Laboratory, \\ John Innes Centre, Colney Lane, Norwich NR4 7UH, Norfolk, U.K.
}

Submitted 14 December 2008. Accepted 29 April 2009.

\begin{abstract}
The tomato $C f-9$ and $C f-9 B$ genes both confer resistance to the leaf mold fungus Cladosporium fulvum but only $C f-9$ confers seedling resistance and recognizes the avirulence (Avr) protein Avr9 produced by $C$. fulvum. Using domain swaps, leucine-rich repeats (LRR) 5 to 15 of $\mathrm{Cf}-9$ were shown to be required for $C f$-9-specific resistance to $C$. $f u l$ vum in tomato, and the entire N-terminus up to LRR15 of Cf-9B was shown to be required for $C f-9 B$-specific resistance. Finer domain swaps showed that nine amino-acid differences in LRR 13 to 15 provided sufficient Cf-9-specific residues in a Cf-9B context for recognition of Avr9 in Nicotiana tabacum or sufficient $\mathrm{Cf}-9 \mathrm{~B}$ residues in a $\mathrm{Cf}-9$ context for a novel necrotic response caused by the expression of $\mathrm{Cf}$ 9B in N. benthamiana. The responses conferred by LRR 13 to 15 were enhanced by addition of LRR 10 to 12, and either region of $\mathrm{Cf}-9 \mathrm{~B}$ was found to cause necrosis in $\mathrm{N}$. benthamiana when the other was replaced by $\mathrm{Cf}-9$ sequence in a Cf-9B context. As a consequence, the domain swap with LRR 13 to 15 of $\mathrm{Cf}-9$ in a Cf-9B context gained the dual ability to recognize Avr9 and cause necrosis in $N$. benthamiana. Intriguingly, two Cf-9B-specific domain swaps gave differing results for necrosis assays in $N$. benthamiana compared with disease resistance assays in transgenic tomato. The different domain requirements in these two cases suggest that the two assays detect unrelated ligands or detect related ligands in slightly different ways. A heat-sensitive necrosis-inducing factor present in $N$. benthamiana intercellular washing fluids was found to cause a necrotic response in N. tabacum plants carrying $H c r 9-9 A, C f-9 B$, and $C f-9$ but not in plants carrying only $C f-9$. We postulate that this necrosis-inducing factor is recognized by $\mathrm{Cf}-9 \mathrm{~B}$ either directly as a ligand or indirectly as a regulator of Cf-9B autoactivity.
\end{abstract}

A. Chakrabarti and S. N. Panter contributed equally to the work reported in this article.

Corresponding author: D. A. Jones; Telephone: +612 6125 4192; Fax: +612 6125 4331; E-mail: david.jones@anu.edu.au

Current address of A. Chakrabarti: CSIRO Plant Industry, GPO Box 1600, Canberra ACT 2601, Australia.

Current address of S. N. Panter: Biosciences Research Division, Department of Primary Industries, Victorian AgriBiosciences Centre, La Trobe University R\&D Park, 1 Park Drive, Bundoora VIC 3083, Australia.

*The $\boldsymbol{e}$-Xtra logo stands for "electronic extra" and indicates that supplementary material including two figures and additional results and experimental procedures is published online.
Plant genes conferring race-specific resistance to plant pathogens have been studied and used productively for much of the last century with little understanding of how they function at the molecular level. The relatively recent isolation and sequencing of a number of disease resistance genes has paved the way for molecular studies that are now beginning to unravel the complexity of the recognition and signaling processes involved in triggering plant defense responses. An example is the tomato $C f-9$ gene, which confers resistance to races of the leaf-mold fungus Cladosporium fulvum that carry the avirulence (Avr) gene $A v r 9$. Sequence analysis predicted that $C f-9$ encodes a type I, membrane-anchored glycoprotein with a large, extracytosolic, leucine-rich-repeat (LRR) domain and a small cytosolic tail (Jones et al. 1994), and this prediction has been confirmed by biochemical analysis (Benghezal et al. 2000; Piedras et al. 2000). Cf-9 is the founding member of a class of LRR receptor-like resistance proteins, which include the tomato Cf-2 (Dixon et al. 1996), Cf-4 (Thomas et al. 1997), Cf-4E (Takken et al. 1999), Cf-5 (Dixon et al. 1998), Cf-9B (Panter et al. 2002), and 9DC (Van der Hoorn et al. 2001a) proteins conferring resistance to C. fulvum; the tomato $\mathrm{Ve} 1$ and Ve2 proteins conferring resistance to the vascular wilt fungus Verticillium dahliae (Kawchuk et al. 2001); and the apple HcrVf2 protein conferring resistance to the apple scab fungus Venturia inaequalis (Belfanti et al. 2004).

The Cf-9 protein has the hallmarks of a receptor but lacks a signaling domain such as the cytosolic, protein-kinase domain found in the rice Xa21 disease-resistance protein, an LRR receptor-like kinase that otherwise closely resembles Cf-9 (Song et al. 1995). Domain-swapping and gene-shuffling experiments between Cf- 9 and $\mathrm{Cf}-4$ have identified the N-terminal portion of the extracytosolic LRR domain of these proteins as critical for Avr recognition (Van der Hoorn et al. 2001b; Wulff et al. 2001). However, binding studies have been unable to demonstrate binding between the Avr9 peptide and the Cf-9 protein (Luderer et al. 2001) but, instead, show that Avr9 is able to bind to a cell-surface receptor on all solanaceous plants regardless of the presence or absence of $C f-9$ (KoomanGersmann et al. 1996). These two observations suggest that the interaction between Cf-9 and Avr9 may involve other components that mediate the interaction.

$C f-9$ is a member of a large multigene family with at least three clusters of tandemly duplicated paralogous genes residing on the short arm of chromosome 1 (Parniske et al. 1999). $C f-9$ is the central member in a cluster of five paralogues, of which the other four are designated Hcr9-9A, Hcr9-9B, 
Hcr9-9D, and Hcr9-9E (Parniske et al. 1997). Cf-9 confers seedling and mature-plant resistance to $C$. fulvum, whereas $H c r 9-9 B$, now designated $C f-9 B$, protects fruiting plants but not vegetative plants from infection by $C$. fulvum (Panter et al. 2002). Thus, Cf-9 and Cf-9B provide an opportunity to study the basis for mature plant versus seedling resistance in a relatively well-characterized plant-pathogen interaction. A previous study has shown that the observed difference in resistance cannot be explained by a temporal difference in resistance gene expression but might be explained by a temporal difference in the expression of proteins mediating elicitor recognition (Panter et al. 2002).

This article describes domain-swapping experiments that aimed to identify regions of Cf- 9 and Cf-9B involved in elicitor recognition. $\mathrm{Cf}-9$ and $\mathrm{Cf}-9 \mathrm{~B}$ show $89 \%$ identity and $92 \%$ similarity to one another, with most of the differences confined to the N-terminal halves of the proteins (Fig. 1). Domain swaps between $C f-9$ and $C f-9 B$ were expressed in transgenic tomato to test their ability to confer race-specific resistance to leaf mold and in leaves of transgenic tobacco expressing Avr9 to test for recognition of Avr9. Results showed that the determinants of specific resistance to $C$. fulvum were more localized in Cf-9 compared with Cf-9B. Residues in the central portion of the LRR domain were involved in the recognition of Avr9 by Cf-9 in leaves of Avr9 tobacco; however, a larger region of Cf-9B was involved in a novel chlorotic or necrotic response caused by the expression of Cf-9B in the leaves of Nicotiana benthamiana. Interestingly, a heat-sensitive factor present in the apoplast fluids of $N$. benthamiana caused necrosis in tobacco plants carrying $\mathrm{Cf}-9 \mathrm{~B}$, suggesting that $\mathrm{Cf}-9 \mathrm{~B}-$ dependent necrosis in $N$. benthamiana may involve recognition of this necrosis-inducing factor.

\section{RESULTS}

\section{Residues within LRR 13 to 15 of Cf-9 confer recognition of Avr9 but upstream residues contribute to the strength of the Cf-9 response.}

$C f$ - $9 B$-mediated resistance occurs only in mature plants; therefore, seedlings of transgenic tomato lines containing initial Cf-9B/Cf-9 domain swap constructs were inoculated with C. fulvum race 5 in order to test the encoded proteins for $C f-9$ resistance specificity. These plants were also crossed to SLJ6201B (Avr9) tomato plants to test for Avr9 recognition. The results of these experiments, which showed that recognitional specificity was contained within LRR 5 to 15 of Cf-9 and that the N-terminus or LRR 1 to 5 contributed to the strength of the Cf-9 response, were consistent with those of Wulff and associates (2001) and, therefore, have been published as supplemental data (Supplementary Results).

Cf-9B/Cf-9 domain swap constructs were also tested by transient expression following agroinfiltration of SLJ6201A (Avr9) tobacco (N. tabacum) plants as described previously by Thomas and associates (1999) and Van der Hoorn and associates (1999). In describing these experiments, the names of the domain swap constructs are followed by short parenthetic descriptions of the resulting proteins. For $\mathrm{N}$ - or C-terminal swaps, the proteins contributing to the $\mathrm{N}$ - and $\mathrm{C}$-termini are written on the left and right, respectively, with the number of the LRR containing the junction written as a subscript (e.g., $9 \mathrm{~B}_{5} 9$ has the N-terminus of $\mathrm{Cf}-9 \mathrm{~B}$ fused to the C-terminus of Cf-9 in LRR5). For interstitial swaps, the protein backbone is written on the left and the protein contributing the interstitial segment is written as a subscript along with the numbers of the LRR that define the segment (e.g., $9_{9 \mathrm{~B}: 5-15}$ has LRR 5 to 15 from Cf-9B inserted into Cf-9). Transient expression of the pSLJ9601 (Cf-9), pCBJ178 (9B $\left.{ }_{5} 9\right)$, and pCBJ280 (9 $\left.{ }_{15 / 16} 9 \mathrm{~B}\right)$ constructs caused a necrotic response throughout the infiltrated area of SLJ6201A (Avr9) tobacco leaves, whereas pCBJ177 $\left(9_{9 B: 5-15}\right)$, pCBJ201 $\left(9 B_{15 / 16} 9\right)$ or the binary vector with no insert did not cause necrosis (Figs. 2 and 3). None of the constructs caused necrosis in control tobacco leaves, showing that the necrotic responses were dependent on the presence of Avr9. Consistent with the data obtained for transgenic tomato, these results also showed that recognitional specificity was contained within LRR 5 to 15 of Cf-9.

To map recognitional specificity more precisely, the pCBJ222 (99B:5-9), pCBJ223 (9 $\left.9_{9 \mathrm{~B}: 10-15}\right)$, pCBJ224 (998:13-15 $)$, and pCBJ225 (99B:5-12) swaps were made between LRR5 and 15 of $\mathrm{Cf}-9$, in a protein otherwise consisting of the Cf-9 sequence. Transient expression of pCBJ222 (99B:5-9) gave the same necrotic response in SLJ6201A (Avr9) tobacco leaves as the pSLJ9601 (Cf-9) and pCBJ178 $\left(9 \mathrm{~B}_{5} 9\right)$ constructs (Fig. 2). The pCBJ225 (99B:5-12) construct caused a weaker necrotic response that was seen in a number of replicates of the experiment but not all. The pCBJ223 (998:10-15) and pCBJ224 (99B:13-15) constructs did not cause a necrotic response. These results suggested that amino acid residues in LRR 13 to 15 of Cf- 9 were the minimum requirement for specific, albeit weak, recognition of Avr9 but residues in LRR 10 to 12 of Cf-9 contributed to the severity of necrosis.

To further assess the roles of LRR 10 to 12 and 13 to 15 in Avr9 recognition, constructs pCBJ292 (9B $\left.{ }_{9: 5-15}\right)$, pCBJ 293 $\left(9 B_{9: 10-15}\right)$, and pCBJ294 (9B $\left.{ }_{9: 13-15}\right)$ were made in which LRR 5 to 15,10 to 15 , and 13 to 15 of $\mathrm{Cf}-9 \mathrm{~B}$ were replaced by the corresponding regions of Cf-9, respectively. All three of these domain swaps produced necrosis when expressed transiently in SLJ6201A (Avr9) tobacco (Fig. 3). The necrosis produced by pCBJ292 $\left(9 \mathrm{~B}_{9: 5-15}\right)$ and $\mathrm{pCBJ} 293\left(9 \mathrm{~B}_{9: 10-15}\right)$ was as strong as that produced by $C f-9$ itself, whereas the response of pCBJ294 (9B 9:13-15 $)$ was weaker. Additional constructs pCBJ299 $\left(9 \mathrm{~B}_{9: 5-9}\right)$ and pCBJ300 (9B $\left.{ }_{9: 5-12}\right)$, in which LRR 5 to 9 and 5 to 12 of Cf-9B were replaced by the corresponding regions of Cf-9, failed to cause necrosis in SLJ6201A (Avr9) tobacco (Fig. 3). These results confirmed that residues within LRR 13 to 15 of Cf-9 are sufficient for recognition of Avr9 but residues within LRR 10 to 12 of Cf- 9 contribute to the strength of the response.

\section{Residues in the entire N-terminal half of $\mathrm{Cf}-9 \mathrm{~B}$ are required for $\mathrm{Cf}-9 \mathrm{~B}$-mediated resistance.}

Fruiting tomato plants containing the domain swap constructs were inoculated with $C$. fulvum race 5,9, which lacks a functional $A v r 9$ gene (Marmeisse et al. 1993), to assay $C f$ $9 B$-mediated mature-plant resistance. Eight of nine CBJ142 $\left(9 \mathrm{~B}_{15 / 16} 9\right)$ lines showed various degrees of resistance but all nine CBJ143 (99B:5-15) lines and all six CBJ144 (9B 5 9) lines were susceptible (Fig. 4A). Resistance in CBJ142 (9B (15/16 ) plants was characterized either by no symptoms of infection or by various degrees of chlorotic flecking (Fig. 4A). The abaxial surfaces of leaves on resistant CBJ142 (9B B $\left._{15 / 16} 9\right)$ plants had sparse aerial hyphae, invisible to the naked eye. Underdeveloped aerial hyphae and the occasional patch of sporulation were evident when chlorotic spots corresponding to infection sites were cut from resistant leaves, stained for the detection of fungal hyphae with trypan blue, and viewed microscopically (data not shown). However, this resistance did not prevent the growth of branched hyphae between mesophyll cells. Small but dense patches of aerial hyphae and sporulation were evident on stained leaves of mature plants from three CBJ142 (9B $\left.{ }_{15 / 16} 9\right)$ lines (data not shown), indicating weaker resistance than that of the other five resistant CBJ142 $\left(9 \mathrm{~B}_{15 / 16} 9\right)$ lines. Susceptible transgenic plants and Moneymaker control plants showed widespread aerial 


\section{Cf-9B}

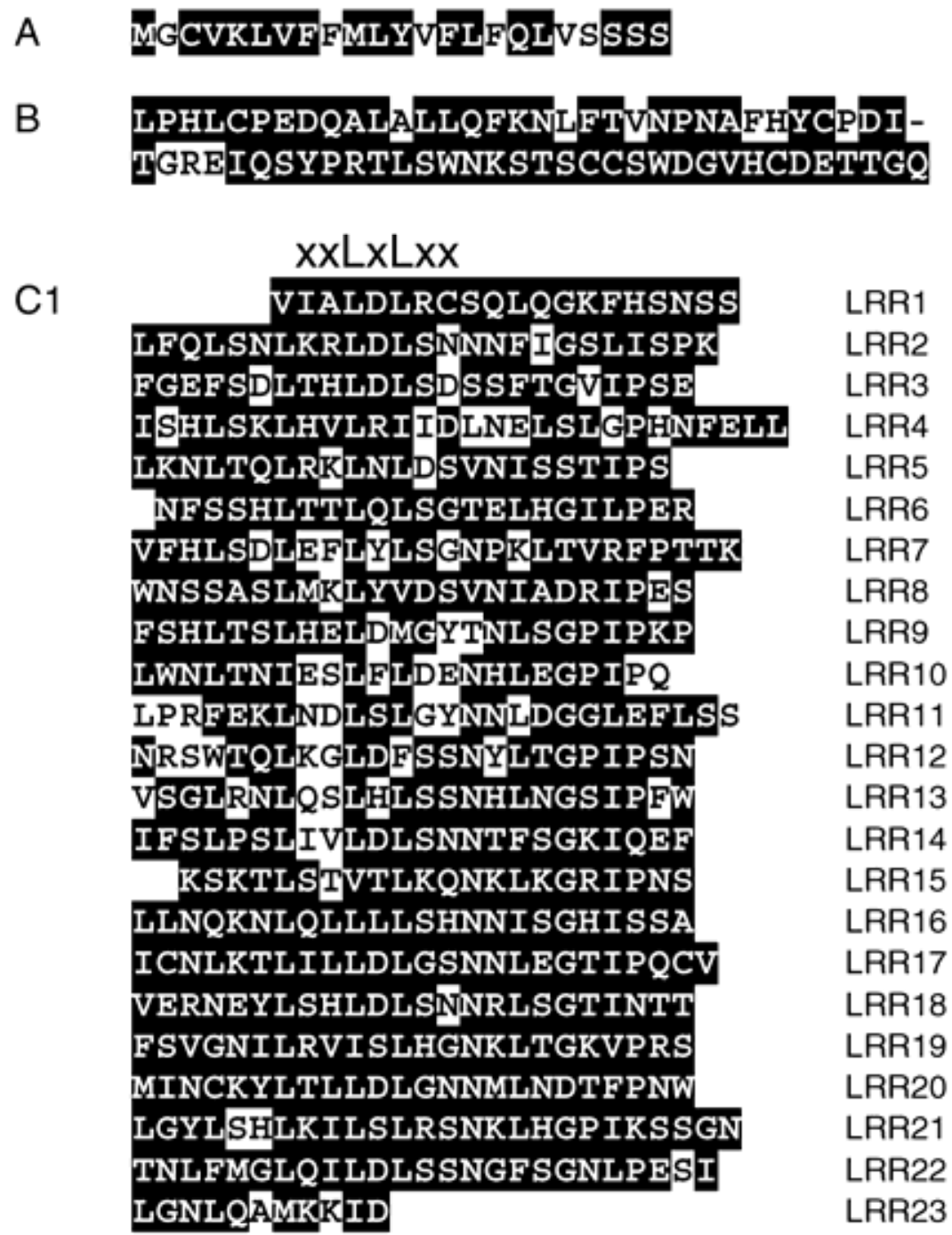

C2 ESTRTPEYISDPYDFYYNYLTTISTKGQDYDS

C3 VRIFNSNMIINLSKNRFEGHIPSI LRR24 IGDLVGLRTLNLSHNALEGHIPAS LRR25

LQNLSVLESLDLSSNKISGEIPQQ LRR26

LASLTFLEVLNLSHNHLVGCIPKG LRR27

D KQFDTFLNSSYQGNDGLRGFPLSKLCGG

E DDQVTTPAELDQEDEEEDSPMIS

F WQGVLMGYGCGLVIGLSVIYIMW

G STQYPALFSRMDLKLEHIITTRMKKHKKRY

Fig. 1. Predicted amino-acid sequence of Cf-9B divided into predicted structural domains and showing amino-acid differences with Cf-9. Domain A is the signal peptide, domain B is the mature N-terminus flanking the leucine-rich repeat (LRR) domain, domain C1 is the first LRR domain, domain C2 is a short intervening domain with no LRR homology, domain C3 is the second LRR domain, domain D is the C-terminal flanking domain, domain E is an acidic, juxtamembrane domain, domain $\mathrm{F}$ is the transmembrane domain and domain $\mathrm{G}$ is a basic, cytosolic, juxtamembrane domain. Amino acid identities with Cf- 9 are highlighted using white text on a black background. The location of the predicted $\beta$-turn/ $\beta$-strand/ $\beta$-turn motif is shown above the residues of the LRR domain by xxLxLxx, where $\mathrm{x}$ represents the various predicted solvent-exposed residues. 

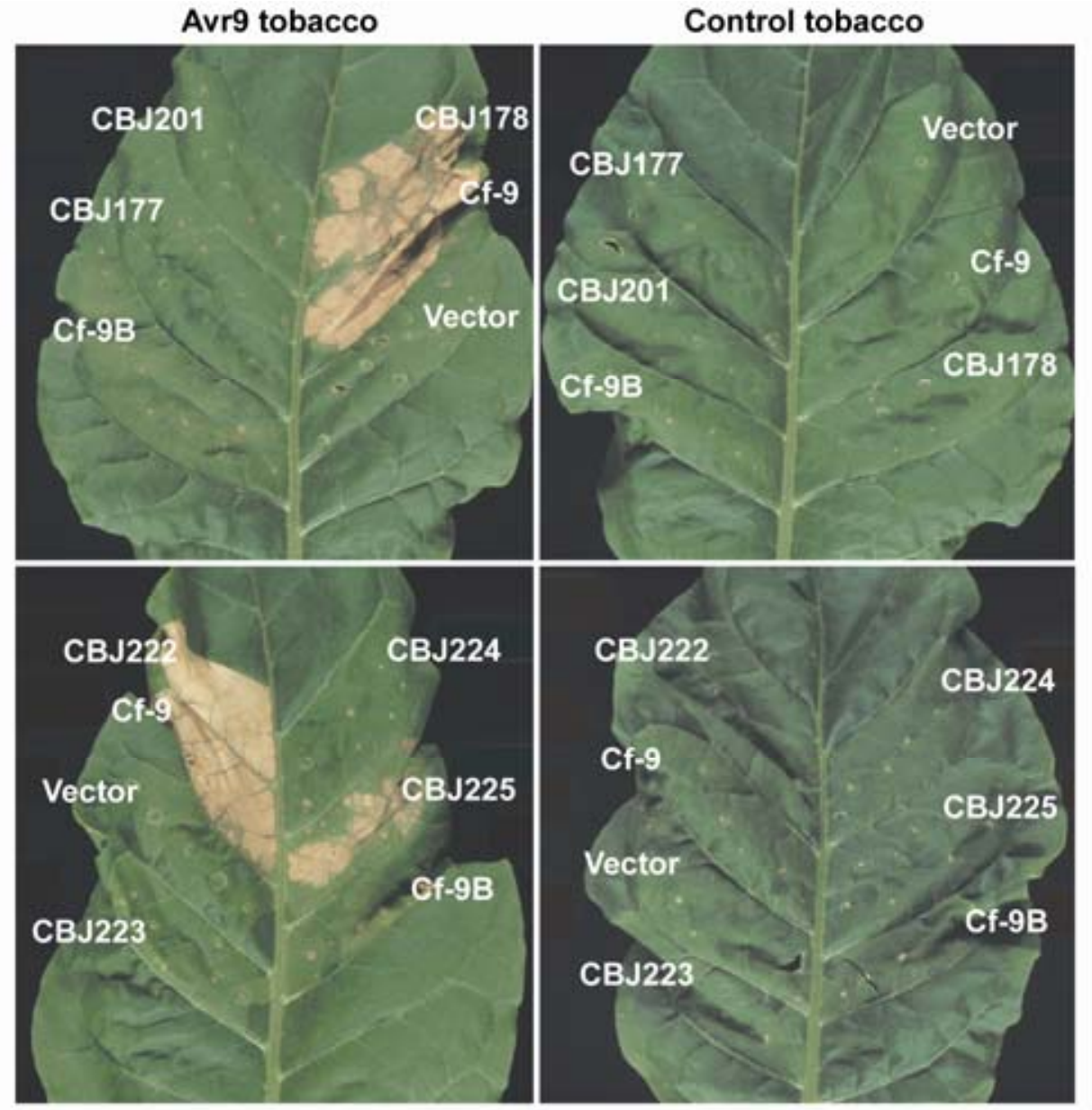

B
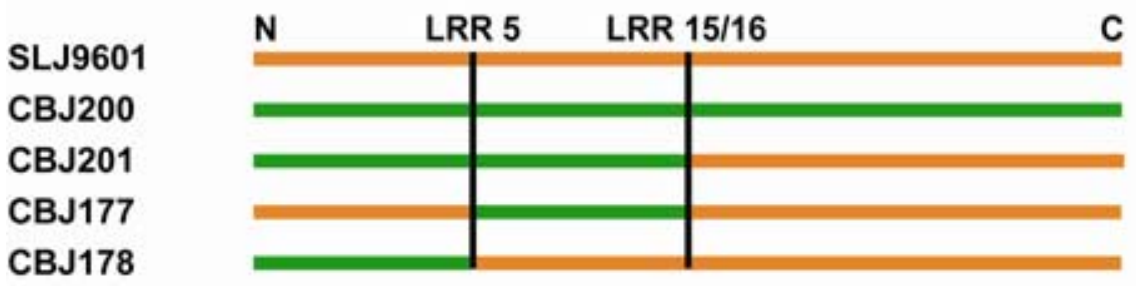

Avr9 tobacco response

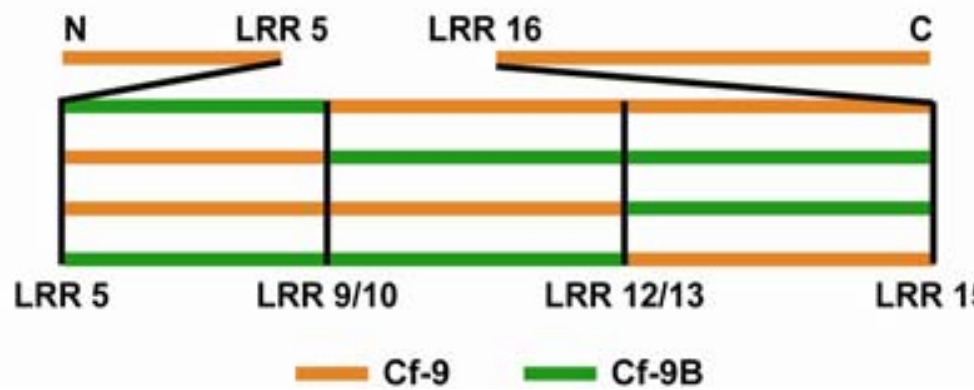

CBJ222

CBJ223

CBJ224

CBJ225
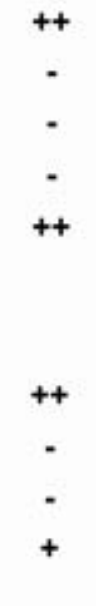

Fig. 2. Analysis of domain swaps constructed in a $C f-9$ context for $C f-9$ specificity by agroinfiltration of Avr9 tobacco leaves. A, Young, fully expanded leaves of budding SLJ6201A (Avr9) tobacco plants (left panels) and untransformed Petit Havana (control) tobacco plants (right panels) were infiltrated with suspensions of Agrobacterium tumefaciens cells containing domain swap or control binary vector constructs. The pSLJ9601 (Cf-9), pCBJ178 (9B 59 ), and pCBJ222 (99B:5-9) constructs caused necrosis throughout the infiltrated leaf panel in Avr9 tobacco leaves, whereas pCBJ225 (99B:5-12) caused less extensive necrosis and pCBJ10 (Vector), pCBJ200 (Cf-9B), pCBJ178 (9B 59 ), pCBJ201 (9B $\left.{ }_{15 / 16} 9\right)$, pCBJ223 (9 $\left.9_{9 \mathrm{~B}: 10-15}\right)$, and pCBJ224 (9 $9_{9 \mathrm{~B}: 13-15}$ ) constructs caused no necrosis. None of the constructs caused necrosis in leaves of control tobacco plants. B, Schematic diagram of the proteins encoded by the various constructs used in this portion of the study and a summary of the results obtained with these constructs following agroinfiltration of pSLJ6201A (Avr9) tobacco. All constructs were expressed under the control of the Cauliflower mosaic virus $35 \mathrm{~S}$ promoter. 
hyphae on the abaxial surfaces of leaves (Fig. 4A; data not shown). Microscopic examination of trypan blue-stained susceptible leaves revealed a dense layer of aerial hyphae at the leaf surface, various degrees of sporulation, and branched hy- phae among the mesophyll cells (data not shown). Overall, these results suggested that residues in the entire $\mathrm{N}$-terminus up to LRR15 of Cf-9B were required for $C f-9 B$-mediated resistance to $C$. fulvum.
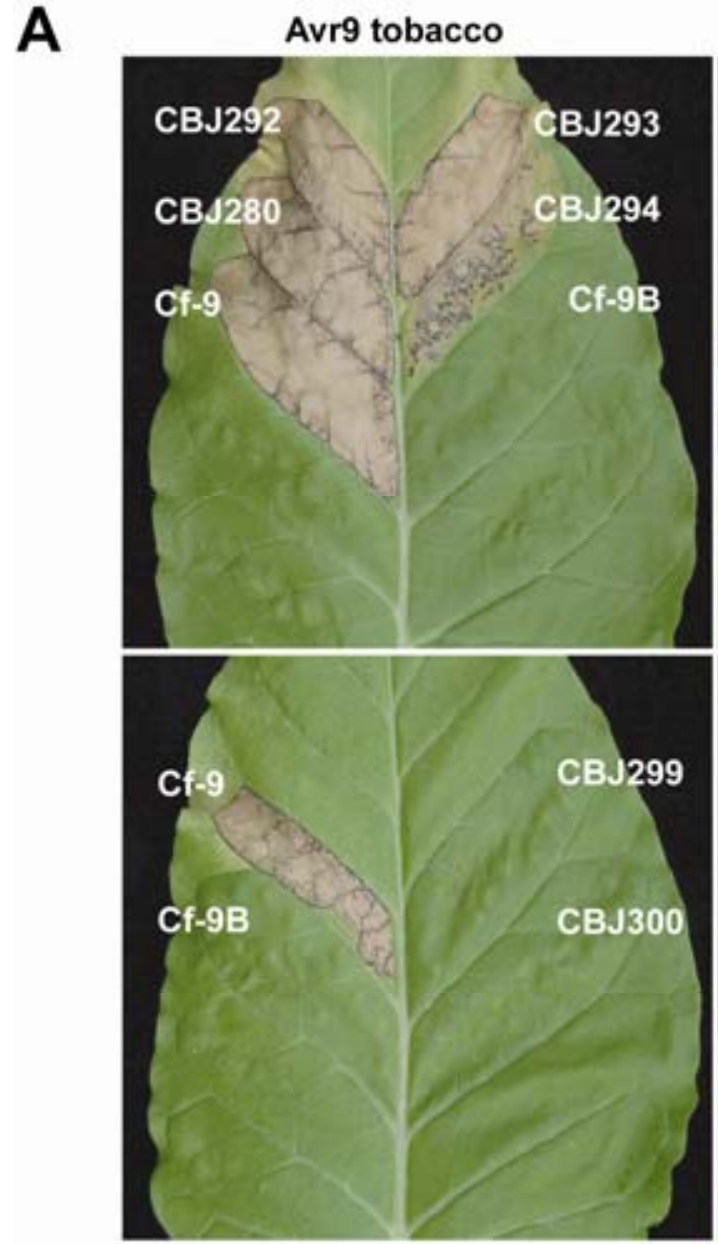

B

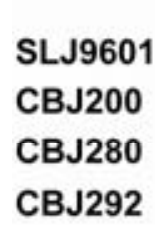

CBJ293

CBJ294

CBJ299

CBJ300

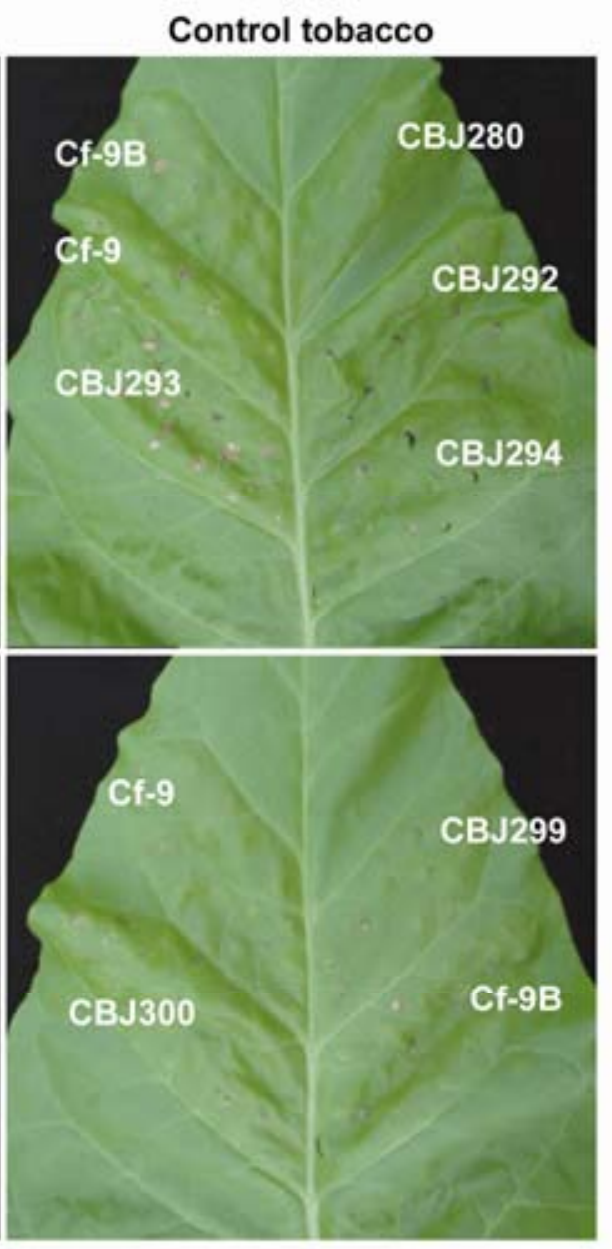

Avr9 tobacco

response

C

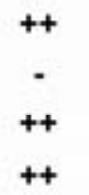

C

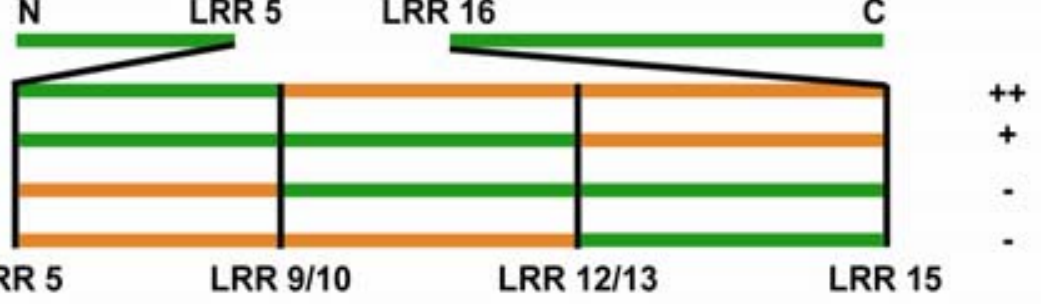

Fig. 3. Analysis of domain swaps constructed in a $C f-9 B$ context for $C f-9$ specificity by agroinfiltration of Avr9 tobacco leaves. A, Young, fully expanded leaves of budding SLJ6201A (Avr9) tobacco plants (left panels) and untransformed Petit Havana (control) tobacco plants (right panels) were infiltrated with suspensions of Agrobacterium tumefaciens cells containing domain swap or control binary vector constructs. The pSLJ9601(Cf-9), pCBJ280 (9 $\left.9_{15 / 16} 9 \mathrm{~B}\right)$,

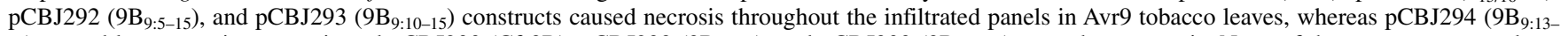
${ }_{15}$ ) caused less extensive necrosis and pCBJ200 (Cf-9B), pCBJ299 (9B $\left.{ }_{9: 5-9}\right)$, and pCBJ300 (9B 9 :5-12) caused no necrosis. None of the constructs caused necrosis in leaves of control tobacco. B, Schematic diagram of the proteins encoded by the various constructs used in this portion of the study and a summary of the results obtained with these constructs following agroinfiltration of pSLJ6201A (Avr9) tobacco. All constructs were expressed under the control of the Cauliflower mosaic virus $35 \mathrm{~S}$ promoter. 

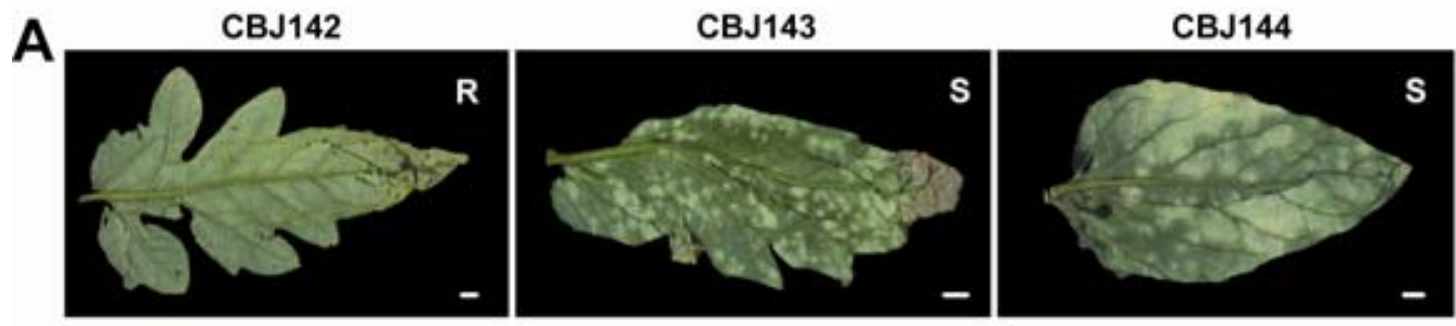

B
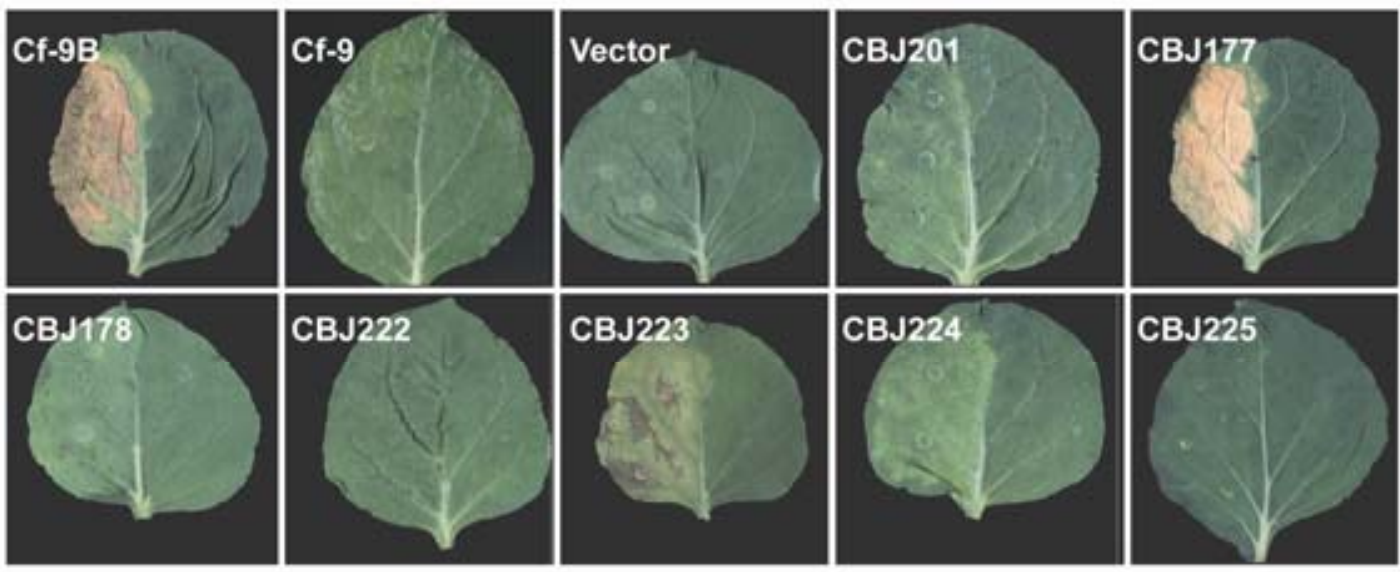

C
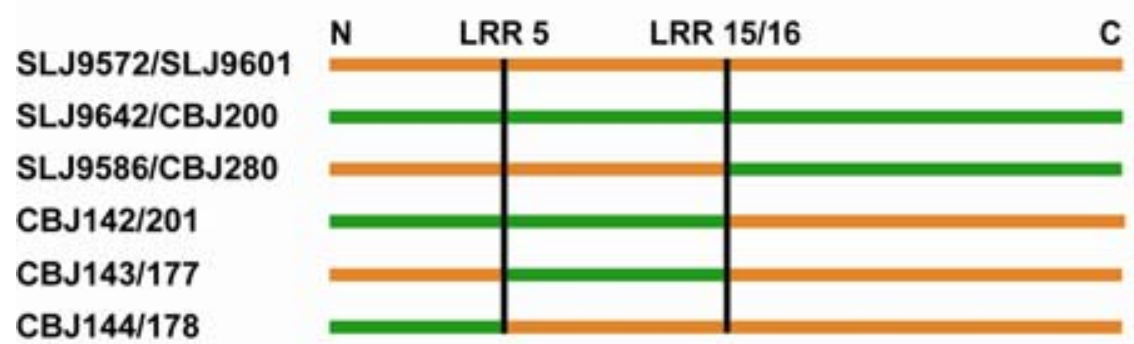

C. fulvum N. benth. race 5,9 response

S
R
R
S
S

CBJ222

CBJ223

CBJ224

CBJ225
LRR 5

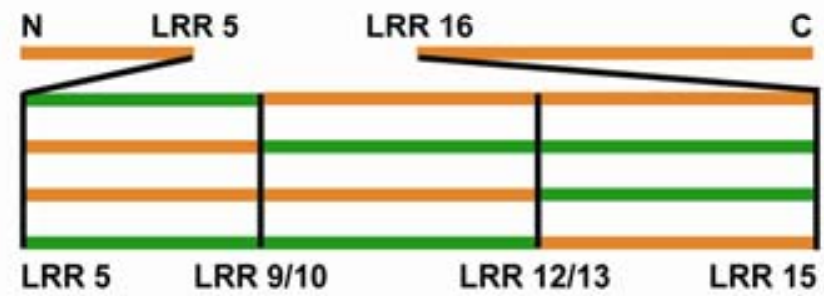

Cf-9

\section{Cf-9B}

Fig. 4. Analysis of domain swaps constructed in a $C f-9$ context for $C f-9 B$-mediated responses following inoculation with Cladosporium fulvum or agroinfiltration of Nicotiana benthamiana leaves. A, Leaves of mature transgenic tomato plants were inoculated with $C$. fulvum race 5,9, which lacks Avr9 but expresses Avr9B, to test for $C f-9 B$ resistance specificity. pCBJ142 $\left(9 \mathrm{~B}_{15 / 16} 9\right)$ plants were resistant $(\mathrm{R})$ and inoculated leaves had barely-visible chlorotic areas. pCBJ143 $\left(9_{9 \mathrm{~B}: 5-15}\right)$ and pCBJ144 $\left(9 \mathrm{~B}_{5} 9\right)$ plants were susceptible $(\mathrm{S})$ and inoculated leaves showed extensive growth of aerial hyphae. The scale bars are equal to $0.5 \mathrm{~cm}$. B, Transient expression of $C f-9 B$ under the control of the Cauliflower mosaic virus (CaMV) $35 \mathrm{~S}$ promoter (pCBJ200) in mature leaves of flowering $N$. benthamiana plants was found to cause spontaneous necrosis. Necrosis was not seen when N. benthamiana leaves were infiltrated with Agrobacterium tumefaciens cells containing a $C f-9$ binary vector construct (SLJ9601) or the empty binary vector pCBJ10 with only the CaMV $35 \mathrm{~S}$ promoter (Vector). Therefore, the Cf-9/Cf-9B domain swap constructs used previously to analyze Cf-9 specificity were used to map regions within the Cf-9B protein that contributed to the necrotic response. Treatment of leaves with the pCBJ177 (99B:5-15) construct resulted in necrosis throughout the infiltrated leaf panel comparable with that observed with the $C f-9 B$ construct. Infiltration of leaves with the pCBJ223 $\left(9_{9 \mathrm{~B}: 10-15}\right)$ and pCBJ224 (9 $\left.9_{9 \mathrm{~B}: 13-15}\right)$ constructs resulted in progressively weaker responses. The pCBJ201 $\left(9 \mathrm{~B}_{15 / 16} 9\right)$ construct was able to cause pale chlorosis in some replicates of the experiment but not in others. The pCBJ178 (9B 59 ), pCBJ222 (998:5-9), and pCBJ225 (99B:5-12) constructs did not cause significant chlorosis or necrosis in N. benthamiana. C, Schematic diagram of the proteins encoded by the various constructs used in this portion of the study and a summary of the results obtained with these constructs following inoculation of mature transgenic tomato plants with $C$. fulvum race 5,9 or following agroinfiltration of $N$. benthamiana. Where pairs of construct names are shown, the lefthand names correspond to constructs under the control of the Cf-9 promoter or, in the case of pCBJ9586, the Cf-9B promoter, which were used for tomato transformation, and the righthand names correspond to constructs under the control of the CaMV $35 \mathrm{~S}$ promoter, which were used for agroinfiltration. All other constructs listed were expressed under the control of the CaMV 35S promoter. 
Residues within LRR 10 to 15 of Cf-9B are required for a necrotic response in $N$. benthamiana.

Van der Hoorn and associates (1999) showed that crude preparations of Avr9 and Avr4 injected into tobacco cv. Petit Havana leaves $24 \mathrm{~h}$ after infiltration with Agrobacterium tumefaciens carrying $C f-9$ and $C f-4$ constructs, respectively, induced necrosis within 7 days. Tobacco leaves expressing Cf-9B were found to be unresponsive following injection of intercellular washing fluids (IF) isolated from mature Moneymaker tomato plants infected with C. fulvum race 5,9. Therefore, agroinfiltration followed by IF injection was tested as a possible means to detect recognition of Avr9B. Transient expression of pCBJ200 $(C f-9 B)$ followed $24 \mathrm{~h}$ later by the injection of tomato IF containing Avr9B was tried without success in leaves of $N$. tabacum cv. Petit Havana but led to necrosis in leaves of $N$. benthamiana that was seen 7 to 14 days after treatment. Subsequently, it was found that this response occurred after agroinfiltration alone and was independent of the tomato IF (Fig. 4B). The necrotic response was specific to $\mathrm{Cf}-9 \mathrm{~B}$, because infiltration with Agrobacterium cells containing pSLJ9601 ( $C f-9)$ or a binary vector without an insert did not cause necrosis in $N$. benthamiana. Agroinfiltration with Cf-9B was found to cause necrosis in $N$. benthamiana plants of all developmental stages rang- ing from seedlings to large mature plants but not in senescent plants (Supplementary Fig. 1).

The molecular determinants of the $C f-9 B$-dependent necrotic response in $N$. benthamiana were investigated using the same Cf-9/9B domain swaps used to investigate Cf-9 specificity. The transient expression of pCBJ200 $(C f-9 B)$ and pCBJ177 $\left(9_{9 \mathrm{~B}: 5-15}\right)$ in $N$. benthamiana resulted in equally strong necrosis; however, transient expression of pCBJ201 $\left(9 \mathrm{~B}_{15 / 16} 9\right)$ caused only pale chlorosis, barely visible against background leaf color, in some replicates of the experiment (Fig. 4B). Hence, there was a discordance between the inability of pCBJ201 $\left(9 B_{15 / 16} 9\right)$ and ability of pCBJ177 (9 $\left.9_{9 \mathrm{~B}: 5-15}\right)$ to cause necrosis in $N$. benthamiana, on the one hand, and the converse ability and inability of the corresponding pCBJ142 $\left(9 \mathrm{~B}_{15 / 16} 9\right)$ and pCBJ143 $\left(9_{9 \mathrm{~B}: 5-15}\right)$ constructs to confer $C f-9 B$-mediated resistance in tomato, on the other (Fig. 4C).

Among the finer domain swaps, transient expression of pCBJ223 (9 $\left.9_{9 \text { B:10-15 }}\right)$ caused a weaker necrotic response in $N$. benthamiana than pCBJ200 $(C f-9 B)$ or pCBJ177 $\left(9_{9 \mathrm{~B}: 5-15}\right)$, and transient expression of pCBJ224 (9 $\left.9_{9 \mathrm{~B}: 13-15}\right)$ led to chlorosis throughout the infiltrated area (Fig. 4B). Infiltration with Agrobacterium cells containing the pCBJ178 $\left(9 \mathrm{~B}_{5} 9\right)$, pCBJ222 $\left(9_{9 \mathrm{~B}: 5-9}\right)$, or pCBJ225 (9 $\left.9_{9 \mathrm{~B}: 5-12}\right)$ constructs did not lead to necro-
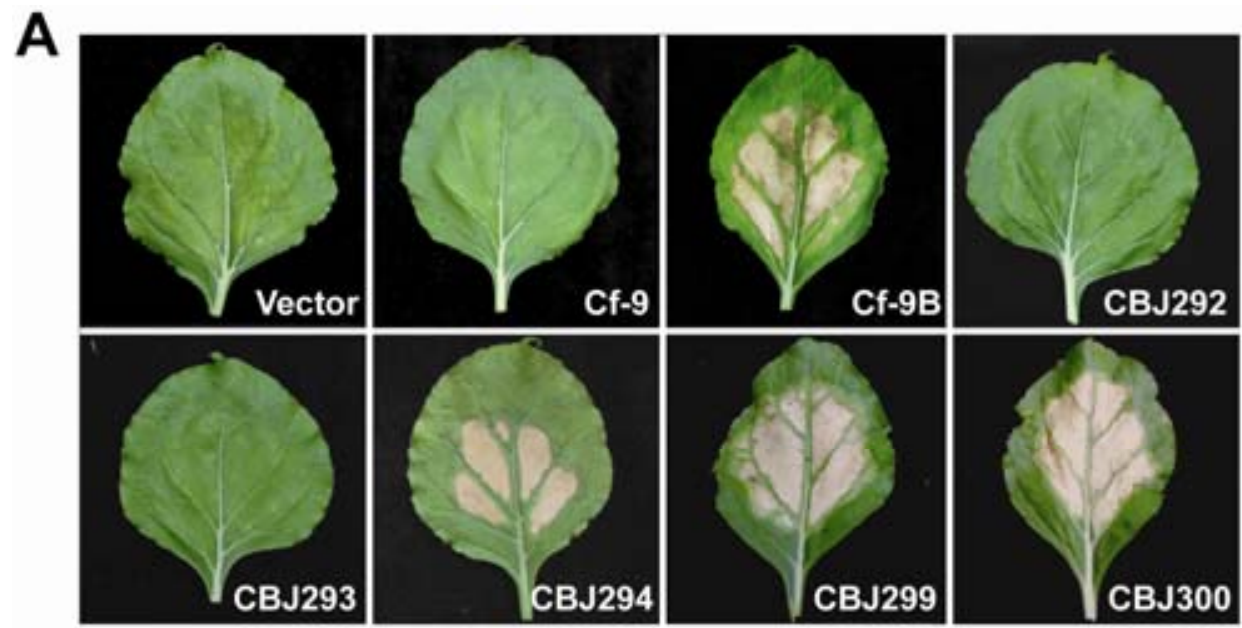

B
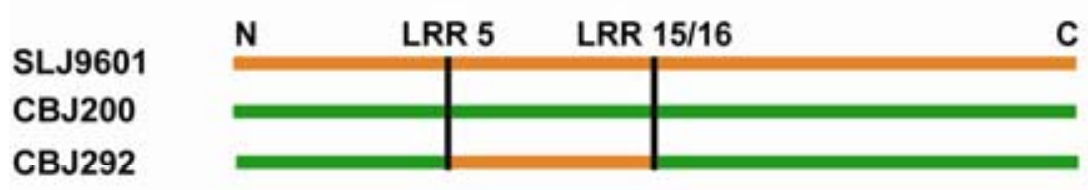

N. benth. response

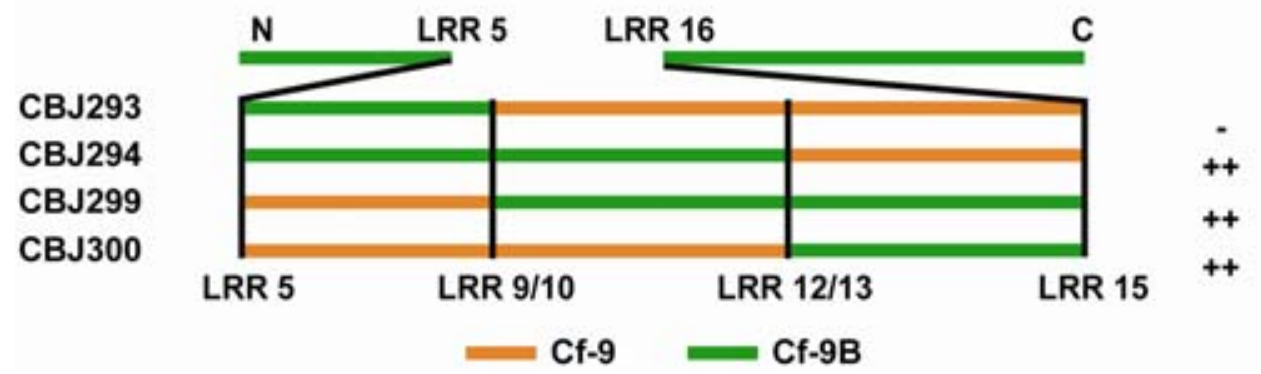

Fig. 5. Analysis of domain swaps constructed in a $C f-9 B$ context for $C f$ - $9 B$-mediated responses following agroinfiltration of Nicotiana benthamiana leaves. A, Mature leaves of flowering $N$. benthamiana plants were infiltrated with suspensions of Agrobacterium tumefaciens cells containing domain swap or control binary vector constructs. pCBJ200 (Cf-9B), pCBJ294 (9B 9:13-15), pCBJ299 (9B 9:5-9), and pCBJ300 (9B $\left.{ }_{9: 5-12}\right)$ caused necrosis in N. benthamiana leaves, whereas CBJ10 (Vector), pSLJ9601 (Cf-9), pCBJ292 (9B $9: 5-15)$, and pCBJ293 (9B $9: 10-15)$ caused no necrosis. B, Schematic diagram of the proteins encoded by the various constructs used in this portion of the study and a summary of the results obtained with these constructs following agroinfiltration of $N$. benthamiana. All constructs were expressed under the control of the Cauliflower mosaic virus 35S promoter. 
sis or chlorosis. Overall, these results suggested that LRR 13 to 15 of $\mathrm{Cf}-9 \mathrm{~B}$ were the minimum required in a Cf-9 context for a response in $N$. benthamiana but residues in LRR 5 to 12 of Cf-9B were needed to enhance this response.

Consistent with these results, pCBJ299 (9B $\left.{ }_{9: 5-9}\right)$ and pCBJ300 (9B $\left.{ }_{9: 5-12}\right)$ caused necrosis in $N$. benthamiana but pCBJ280 (9 $\left.9_{15 / 16} 9 \mathrm{~B}\right)$, pCBJ292 (9B 9:5-15 $)$, and pCBJ 293 (9B 9:10- $_{10}$ ${ }_{15}$ ) did not (Fig. 5; results not shown). However, transient expression of pCBJ294 (9B $9: 13-15)$ also caused necrosis comparable with that caused by Cf-9B (Fig. 5). This suggests that, in a Cf-9B context, residues within either LRR 10 to 12 or 13 to 15 were sufficient to cause necrosis in $N$. benthamiana. Domain swaps caused a stronger response in $N$. benthamiana if the $\mathrm{N}$ terminus up to LRR5 and the C- terminus from LRR15 were derived from Cf-9B (Fig. 5) rather than Cf-9 (Fig. 4), suggesting that residues in these regions also contribute to the magnitude of the response in $N$. benthamiana.

\section{Cf-9B-dependent necrosis is caused}

by a heat-sensitive component of $N$. benthamiana IF.

The necrosis triggered by Cf-9B when it is expressed in $N$. benthamiana could have been caused by autoactivation or by recognition of a protein expressed in the leaves of $N$. benthamiana plants. To test the latter hypothesis, IF was extracted from the mature leaves of flowering $N$. benthamiana plants and injected into the leaves of budding 2/9-34 tobacco plants (Hammond-Kosack et al. 1998), which contain Hcr9-9A, Cf-9B, and $C f-9$. N. benthamiana IF caused a reproducible chlorotic response in 2/9-34 (Hcr9-9A, Cf-9B, and $C f-9$ ) plants but not in control cv. Petit Havana tobacco plants (Fig. 6). This result suggested that a recognition event could have occurred between a soluble component in $N$. benthamiana IF and either Hcr9-9A, Cf-9B, or Cf-9.

The heat stability of the active molecule in $N$. benthamiana IF was tested by boiling the IF for $10 \mathrm{~min}$, separating the solids by centrifugation $(13,500 \times g, 2 \mathrm{~min}$, ambient temperature), and injecting the cooled supernatant into tobacco leaves. The boiled IF was no longer capable of causing chlorosis in 2/9-34 tobacco leaves, indicating that the active component in the $N$. benthamiana IF was heat sensitive (Fig. 6). However, untreated IF was still able to cause chlorosis in 2/9-34 (Hcr9$9 A, C f-9 B$, and $C f-9)$ tobacco leaves even after extended storage at $-20^{\circ} \mathrm{C}$.

When $N$. benthamiana IF was concentrated fourfold by freeze drying and redissolving in $20 \mathrm{mM}$ phosphate buffer $(\mathrm{pH}$ 5.5) containing $100 \mathrm{mM} \mathrm{NaCl}$, it was found to cause necrosis rather than chlorosis in 2/9-34 (Hcr9-9A, Cf-9B, and Cf-9) tobacco leaves (Fig. 6). Concentrated IF from $N$. benthamiana plants of all developmental stages, ranging from seedlings to senescent plants, was found to cause necrosis in Cf-9B tobacco plants (Supplementary Fig. 2).

The induction of necrosis in $N$. benthamiana by Cf-9B but not Cf-9 suggested that the response of 2/9-34 (Hcr9-9A, Cf-9B, and $C f-9$ ) tobacco plants to $N$. benthamiana IF was more likely to have been mediated by Cf-9B than Hcr9-9A or Cf-9. To test this hypothesis, $H c r 9-9 A, C f-9 B$, and $C f-9$ were each transiently expressed in $N$. benthamiana under the control of the Cauliflower mosaic virus (CaMV) 35S promoter. Surprisingly, both Hcr9-9A and $C f-9 B$ caused necrosis in $N$. benthamiana (Fig. 7A).

\section{DISCUSSION}

The N-termini of $\mathrm{Cf}-9$ and $\mathrm{Cf}-9 \mathrm{~B}$ play a role in $\mathrm{Cf}-9$ and $\mathrm{Cf}-9 \mathrm{~B}$ function in tomato.

Consistent with the Cf-4/Cf-9 domain swap data of Wulff and associates (2001) and Van der Hoorn and associates (2001b), the Cf-9/Cf-9B domain swap data showed that poly- morphic amino acid residues in LRR 5 to 15 of Cf-9 were the determinants of Cf- 9 specificity but polymorphic residues in the mature N-terminus (domain B) or LRR 1 to 5 of Cf-9 made a contribution to the magnitude of the plant's response to Avr9. Whereas our domain swaps did not enable a distinction to be made between the contributions of domain B and LRR 1 to 5 of Cf-9, the Cf-4/Cf-9 domain swaps indicated that domain $\mathrm{B}$ of $\mathrm{Cf}-9$ can be replaced by the corresponding region of Cf-4 with no effect on Cf-9 function; however, the additional replacement of LRR 1 to 3 and part of LRR4 caused a reduction in Cf-9 function (Wulff et al. 2001). In contrast to Cf-9, the entire region between the mature $\mathrm{N}$-terminus and LRR15 of Cf-9B (including domain B and LRR 1 to 5) was found to be required for Cf-9B-mediated resistance to $C$. fulvum, indicating a role for domain B or LRR 1 to 5 in Cf-9B function.

Cf-4/Cf-9 domain swaps have shown that a deletion in domain $\mathrm{B}$ of 10 amino acids relative to $\mathrm{Cf}-9$ is important for $\mathrm{Cf}-4$ function but the 4 amino acid differences in domain $\mathrm{B}$ are not required (Van der Hoorn et al. 2001b; Wulff et al. 2001). In contrast, Cf-9B has a deletion in domain B of only one amino acid residue relative to $\mathrm{Cf}-9$ but carries nine amino acid differences in domain B. Therefore, it is possible that polymorphic residues in domain $\mathrm{B}$ may play a more important role in $\mathrm{Cf}-9 \mathrm{~B}$ function than they do for Cf-4.

\section{A smaller region of $\mathrm{Cf}-9$ is involved in recognition of Avr9 than previously described.}

This study, based on Cf-9/9B domain swaps, has identified a smaller region of Cf-9 capable of Avr9 recognition in tobacco agroinfiltration assays than did previous studies based on $\mathrm{Cf}$ -

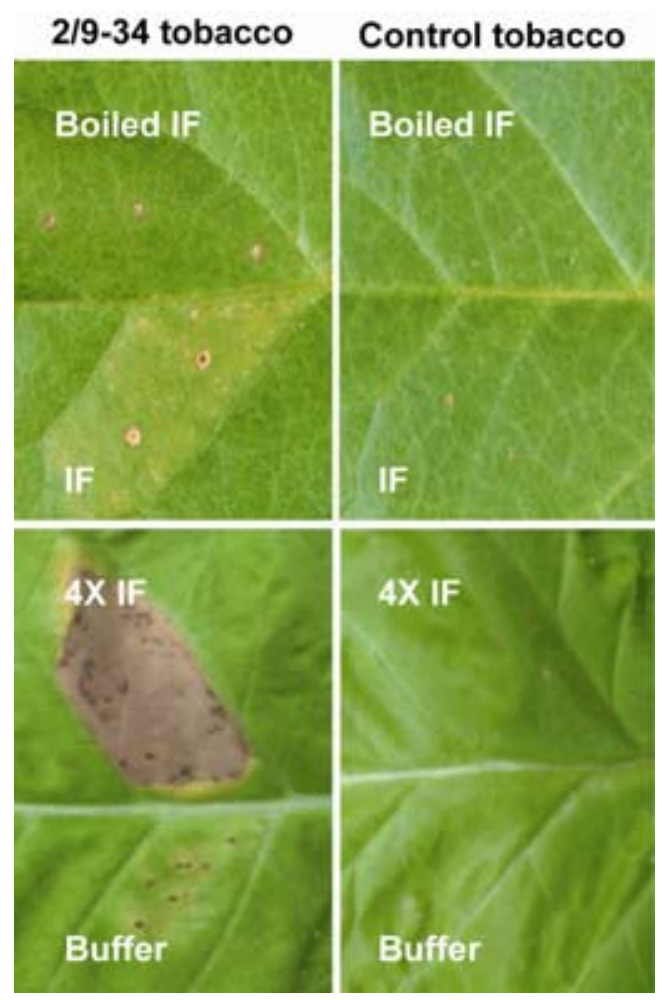

Fig. 6. Analysis of necrosis induction. Intercellular washing fluid (IF) was collected from leaves of mature Nicotiana benthamiana plants and infiltrated either untreated or after boiling into young, fully expanded leaves of budding 2/9-34 (Hcr9-9A, Cf-9B, and Cf-9) and Petit Havana (control) tobacco plants. Untreated IF but not boiled IF caused chlorosis in leaves of 2/9-34 tobacco plants. A fourfold concentration of IF (4XIF) in $20 \mathrm{mM}$ phosphate, $\mathrm{pH} 5.5,100 \mathrm{mM} \mathrm{NaCl}$ (Buffer) caused strong necrosis, whereas buffer alone caused no necrosis. No necrosis was observed in leaves of control tobacco plants. 
4/9 domain swaps, which did not enable better resolution than LRR 6 to 17 of Cf-9 in one study (Van der Hoorn et al. 2001b) or LRR 8 to 18 in another (Wulff et al. 2001). Cf-4/9 geneshuffling experiments showed variant residues in LRR 8 and 9 to be dispensable but they did not enable better resolution than LRR 10 to 18 of Cf-9. Moreover, the constructs with the minimal regions necessary for Avr9 recognition, LRR 6 to 18 in one study (Van der Hoorn et al. 2001b) and LRR 10 to 18 in
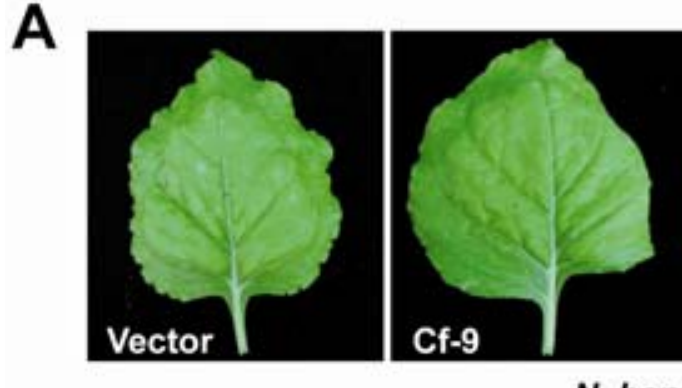

$N$. benthamiana
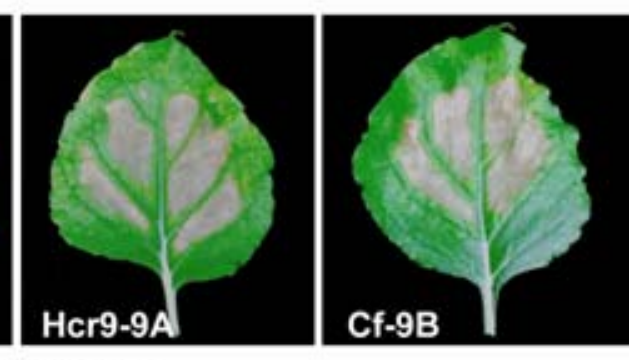

Cf-9B

\begin{tabular}{|c|c|c|c|}
\hline \multirow[t]{2}{*}{ LRR } & 89 & 10 & 1415 \\
\hline & $\mathbf{x} \quad \boldsymbol{x} x \boldsymbol{x x} \quad \mathrm{x} \quad \mathbf{x x x}$ & $\mathbf{x} \times \mathbf{x x x}$ & $\mathbf{x} \times \mathbf{x} \times \mathbf{x}$ \\
\hline \multirow[t]{3}{*}{ Residue } & 222222222222 & 33333333333333333333 & 333334444 \\
\hline & 044444568999 & 11122333333444456667 & 788880113 \\
\hline & 102358193589 & 67912012349045872360 & 936791013 \\
\hline$C f-9$ & BNOSHVQIKYRC & VEHGDSHFTIKRVNEFI & IQECYSVEA \\
\hline Her 9-9DC & QKEDYT & ESDRYPQLPI & IQECY \\
\hline Her9-NLOC & EYRS & ESFD & \\
\hline Her $9-9 \mathrm{E}$ & EDYT & ESDD & \\
\hline Her 9-4C & KEDYT & ESD & \\
\hline HCr9-NLOD & JEHYT & ESFI & \\
\hline $\begin{array}{l}\text { Her 9-9D } \\
\text { Her 9-4E }\end{array}$ & EBDYT & ESDF & A \\
\hline Her 9-NLOE & --- & & VI \\
\hline$C f-4$ & EYRS & $\overline{\mathrm{VEDNN}}--------$ & VRQI \\
\hline Her 9-4A & QKEDYT & $\overline{E S F D}$ & VRQLHSVVT \\
\hline Her $9-9 A$ & GQKEDYT & QLPRN & VRQLHSVVT \\
\hline Hcr9-SCOA & DDEFHGQKEDYT & ESFDDPQLPRNDGYLSEIFY & VRQLHSVVT \\
\hline$C f-9 B$ & DDEFYGKKEDYT & ESFDËPQLPRNDGYLSKGFY & VRQSHFIVT \\
\hline \multirow[t]{2}{*}{ LRR } & 89 & 10 & 1415 \\
\hline & $\mathbf{x} \quad \operatorname{xxxx} \quad x \quad \mathbf{x x x}$ & $\mathbf{x} \times \mathbf{x x}$ & $\mathbf{x x x} \times \mathbf{x x}$ \\
\hline \multirow[t]{3}{*}{ Residue } & 222222222222 & 33333333333333333333 & 333334444 \\
\hline & 034444568999 & 11122233333344456667 & 888890113 \\
\hline & 091247082478 & 56801901238934764582 & 158913235 \\
\hline$C f-9 B$ & DDEFYGKKEDYT & ESFD FPQLPRNDGYLSKGFY & VRQSHEIVT \\
\hline Her $9-9 A$ & DDEFECOKBDYT & BSFD PPQLPRNDGYLSE IFY & VRQIHSVVT \\
\hline Hcr 9-9D & YDEEDY OKBDYT & RYPQLPIKK & VRQSY SIVA \\
\hline Her $9-4 \mathrm{E}$ & YT & & VRQSYSRSI \\
\hline Her 9-NLOC & ENSSDFONEYRS & VLS & \\
\hline Cf-4 & EDEFDS KKYRS & 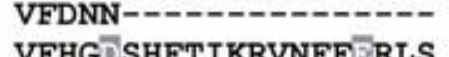 & RQIW' \\
\hline $\mathrm{CI}-\mathrm{y}$ & ENQSHVOTKYRC & VEHGDSH & YSVEA \\
\hline
\end{tabular}

Fig. 7. Variation between members of the Hcr9 family for necrosis induction in Nicotiana benthamiana, Avr9 recognition, and amino acid residues at positions in leucine-rich repeats (LRR) 5-15 that differ between Cf-9 and Cf-9B. A, Hcr9-9A, $C f-9 B$, and $C f-9$ were each transiently expressed in the leaves of $N$. benthamiana. Both Hcr9-9A and $C f-9 B$ but not $C f-9$ caused necrosis in $N$. benthamiana. All constructs were expressed under the control of the Cauliflower mosaic virus $35 \mathrm{~S}$ promoter in the binary vector pCBJ306 (Vector). B and C, Alignments are shown of predicted Hcr9 amino acid residues corresponding to those involved in Cf-9B/Cf-9 domain swaps and differing between Cf-9 and Cf-9B. The three subregions involved in domain swaps within LRR 5 to 15 are shown separated by gaps in the alignments. LRR repeat number and residues corresponding to predicted solvent exposed residues of the xxLxLxx $\beta$ strand/ $\beta$ turn motif are indicated above the alignment by x. Dashes indicate deletions in individual sequences relative to the others. B, Alignment of sequences that have been tested for Avr9 recognition. Cf-9 and Hcr9-9DC are able to recognize Avr9 but the other Hcr9 proteins shown (including Cf-4 and Cf-9B) are not. Residues shared between Cf-9 and other Hcr9 proteins are highlighted in black. Residues shared by Cf-9 and Hcr9-9DC and no more than one other Hcr9 are highlighted in red. Residues unique to Cf- 9 are highlighted in green. Amino acid residue numbers for Cf- 9 are shown above the alignment. C, Alignment of sequences that have been tested in the present study (see A and B) or by Wulff and associates (2004) for autoactivation in N. benthamiana. Cf-9B and Hcr99A are autoactive in $N$. benthamiana but the other Hcr9 proteins shown (including Cf-4 and Cf-9) are not. Residues shared between Cf-9B and the other Hcr9 proteins are highlighted in black. Residues shared between Hcr9-9A and the other Hcr9 proteins but not shared with Cf-9B are highlighted in gray. Residues shared by Cf-9B and Hcr9-9A and no other Hcr9 are highlighted in red. Residues unique to either Cf-9B or Hcr9-9A are highlighted in green and residues unique to both $\mathrm{Cf}-9 \mathrm{~B}$ and $\mathrm{Hcr} 9-9 \mathrm{~A}$ are highlighted in yellow. Amino acid residue numbers for $\mathrm{Cf}-9 \mathrm{~B}$ are shown above the alignment. 
the other (Wulff et al. 2001), both gave a significantly reduced response compared with full-length Cf-9 in tobacco agroinfiltration experiments. In contrast, the Cf-9/9B swaps in our study showed that LRR 13 to 15 of Cf- 9 were sufficient for a basal response to Avr9 and that LRR 10 to 15 were sufficient to give a response equivalent to that of full-length Cf-9 in tobacco agroinfiltration experiments. This greater apparent resolution of Cf-9/9B domain swaps could be due, in part, to greater similarity between the recognition domains of Cf-9 and Cf-9B compared with that between Cf- 9 and Cf- 4 at both the gross structural level (Cf-4 differs from $\mathrm{Cf}-9$ by two substantial deletions) and the sequence level (84\% identity and $88 \%$ similarity versus $72 \%$ identity and $78 \%$ similarity from the mature $\mathrm{N}$ terminus to LRR15). Besides affecting ligand binding directly, domain swapping or gene shuffling can also affect the structural context of the ligand binding site or the capacity for signal transduction in response to ligand binding. We postulate that the greater the similarity between the parental sequences, the more likely the polymorphic residues identified as important for recognition by domain swapping or gene shuffling will reflect residues important for ligand binding rather than structural context or signaling capacity.

Van der Hoorn and associates (2001a) discovered a natural domain swap that arose by recombination between $C f-9$ and Hcr9-9D in wild populations of Lycopersicon pimpinellifolium. This chimeric gene, termed 9DC, contains the Hcr9$9 D$ sequence encoding the $\mathrm{N}$-terminus through to the end of LRR11, with the remainder of the gene composed of $C f-9$ sequence. Necrosis was observed after leaflets of L. pimpinellifolium plants containing the $9 D C$ gene were injected with purified Avr9, or after the $9 D C$ and $A v r 9$ genes were transiently coexpressed in tobacco leaves, indicating that amino acid residues between LRR12 and the C-terminus determine Cf- 9 specificity relative to Hcr9-9D (Van der Hoorn et al. 2001a). This is consistent with the results from the Cf-9B/Cf-9 domain swaps, and may likewise reflect the greater similarity between Cf-9 and Hcr9-9D (83\% similarity and $88 \%$ similarity) than between Cf-9 and Cf-4. However, although greater similarity between progenitor sequences may provide better resolution of polymorphic residues involved in recognitional specificity, it may also exclude nonpolymorphic residues that are important for ligand binding. Nevertheless, such residues must be insufficient by themselves for ligand binding or, if sufficient, do not provide the critical ligand contacts necessary to trigger a response.

\section{Cf-9B-dependent necrosis in $N$. benthamiana requires smaller regions of $\mathrm{Cf}-9 \mathrm{~B}$ than resistance to $C$. fulvum.}

Domain swaps containing LRR 10 to 15 and 13 to 15 of Cf$9 \mathrm{~B}$ in a Cf-9 backbone (pCBJ223 and pCBJ224) triggered weak necrosis and chlorosis, respectively, in $N$. benthamiana, and the reciprocal swaps containing LRR 10 to 15 and 13 to 15 of Cf-9 in a Cf-9B backbone (pCBJ293 and pCBJ294) conferred recognition of Avr9 in N. tabacum. This suggested initially that the necrotic or chlorotic response and the recognition of Avr9 were both mediated by the same regions of Cf-9B and Cf-9, respectively. However, when the reciprocal domain swaps were tested for their ability to induce necrosis in N. benthamiana, the domain swap carrying LRR 13 to 15 of Cf-9 in a Cf-9B backbone (pCBJ294) but not that carrying LRR 10 to 15 of Cf-9 (pCBJ293) was found to trigger necrosis. Moreover, a domain swap carrying LRR 5 to 12 of Cf-9 in a Cf-9B backbone (pCBJ300) was also found to trigger necrosis. Together, these results indicate that two regions of Cf-9B, LRR 10 to 12 and 13 to 15 , are each able to trigger necrosis independently of one another in a Cf-9B context, but only LRR 13 to 15 are able to trigger a response in a Cf- 9 context. More- over, the necrotic responses caused by either region in a Cf-9B context was stronger than the necrosis caused by LRR 13 to 15 or 10 to 15 of Cf-9B in a Cf-9 context. This context-dependent difference in necrosis induction is quite dissimilar to the recognition of Avr9, which appeared to be relatively context independent, and indicates the broader involvement of residues in other domains of Cf-9B. Interestingly, the pCBJ294 (9B 9:13-15 $)$ domain swap is able to both recognize Avr9 and trigger necrosis in $N$. benthamiana, suggesting the possible generation of a Cf protein with dual specificity.

\section{Cf-9B-dependent necrosis in $N$. benthamiana requires matching $\mathrm{N}$ - and $\mathrm{C}$-termini.}

The pCBJ201 $\left(9 \mathrm{~B}_{15 / 16} 9\right)$ domain swap containing the N-terminus-LRR15 of Cf-9B caused a very weak response in $N$. benthamiana, whereas the pCBJ200 (Cf-9B) and pCBJ177 (9 $\left.9_{9 \mathrm{~B}: 5-15}\right)$ constructs both caused necrosis in $N$. benthamiana. The latter both contain regions flanking LRR 5 to 15 of $\mathrm{Cf}-9 \mathrm{~B}$ derived from the same resistance protein (i.e., either both Cf$9 \mathrm{~B}$ or both $\mathrm{Cf}-9)$. However, the N-terminal flanking region of pCBJ201 $\left(9 \mathrm{~B}_{15 / 16} 9\right)$ is from $\mathrm{Cf}-9 \mathrm{~B}$ and the $\mathrm{C}$-terminal flanking region from $\mathrm{Cf}-9$. Matching $\mathrm{N}$ - and $\mathrm{C}$-terminal residues from the same resistance protein might be needed for a strong necrotic response if signal activation involves an intra- or intermolecular interaction between the $\mathrm{N}$ - and $\mathrm{C}$-terminal domains of Cf-9B.

\section{The relationship between disease resistance and necrosis induction conferred by Cf-9B.}

The $9 B_{15 / 16} 9$ domain swap conferred mature plant resistance to $C$. fulvum in tomato but caused a very weak response in $N$. benthamiana, whereas the $9_{9 \mathrm{~B}: 5-15}$ domain swap did not confer mature plant resistance to $C$. fulvum in tomato but did cause a strong necrotic response in $N$. benthamiana. Therefore, the relationship between the necrotic or chlorotic response triggered by Cf-9B in $N$. benthamiana and mature plant resistance to $C$. fulvum conferred by $\mathrm{Cf}-9 \mathrm{~B}$ in tomato is unclear. The two responses could be distinct properties of Cf-9B or one could be related to the other. $\mathrm{Cf}-9 \mathrm{~B}$ confers weak resistance to $C$. fulvum and a small loss of Cf-9B function could easily lead to a complete loss of resistance, making it a relatively insensitive indicator of recognitional specificity. In comparison, spontaneous necrosis or chlorosis may be a much more sensitive indicator, enabling smaller regions of $\mathrm{Cf}-9 \mathrm{~B}$ specificity to be identified. This might explain why the $9_{9 \mathrm{~B}: 5-15}$ domain swap could cause necrosis in $N$. benthamiana but could not confer resistance to $C$. fulvum in tomato. However, this does not explain why the $9 \mathrm{~B}_{15 / 16} 9$ domain swap could confer resistance in tomato but could not cause necrosis in $N$. benthamiana. It is possible that recognition of Avr9B in tomato is unrelated to the activation of $\mathrm{Cf}-9 \mathrm{~B}$ signaling in $N$. benthamiana or that the activation of Cf-9B signaling in response to Avr9B does not require matching $\mathrm{N}$ - and $\mathrm{C}$-termini. However, the finding that regions outside of LRR 10 to 15 of Cf-9B, including domain $\mathrm{B}$ or LRR 1 to 5 of Cf-9B, also play a role in necrosis induction mirrors the requirement in tomato for the entire region between the N-terminus and LRR15 of Cf-9B for mature plant resistance to $C$. fulvum, and suggests that the two may be related.

\section{Inferences about residues in $\mathrm{Cf}-9$ and $\mathrm{Cf}-9 \mathrm{~B}$ involved in Avr9 recognition and necrosis induction.}

In this study, variant residues in LRR 13 to 15 of Cf-9 were the minimum required in a $\mathrm{Cf}-9 \mathrm{~B}$ context to confer a basal response to Avr9 in tobacco agroinfiltration assays; however, residues in LRR 10 to 12 were also required to give a response equivalent to that of full-length Cf-9. Similarly, variant residues in LRR 13 to 15 of Cf-9B were the minimum required in 
a Cf-9 context to confer chlorosis in $N$. benthamiana agroinfiltration assays but residues in both LRR 5 to 9 and 10 to 12 were also required to give a necrotic response equivalent to that of full-length Cf-9B. However, variant residues in either LRR 10 to 12 or 13 to 15 were sufficient to cause full necrosis in a Cf-9B context.

There are nine amino acid differences between Cf-9 and Cf9B in LRR 13 to 15,20 in LRR 10 to 12 , and 12 in LRR 5 to 9 (Fig. 7), plus a deletion of three amino acids (RSW) in LRR12 of Cf-9 compared with Cf-9B. However, only six of these differences are unique or nearly unique to Cf-9 and 9DC compared with Hcr9 proteins unable to recognize Avr9; namely, the RSW deletion and five amino acid differences: R363 and L366 located in LRR 12, I379 and C387 located in LRR 13, and A433 located in LRR15 (Fig. 7B). An RSW insertion into LRR12 of Cf-9 had only a slight effect on function despite the introduction of a glycosylation site missing from Cf-9 but present in Cf-9B and other Hcr9 proteins (Van der Hoorn et al. 2005). Wulff and associates (2009) show that C387 and A433 but not $\mathrm{I} 379$ are, indeed, essential for Avr9 recognition in a Cf9B context. However, Wulff and associates (2009) also show that Y389 and E411, which are shared with other Hcr9 proteins proteins, are also required for Avr9 recognition in a Cf-9B context. Unlike L366 and I379, these residues are all predicted to be solvent-exposed residues in the $\beta$-strand/ $\beta$-turn regions of LRR 13 to 15. Similarly, studies of the amino acid residues critical for Avr4 recognition by Cf-4 (Van der Hoorn et al. 2001b), flagellin recognition by FLS2 (Dunning et al. 2007), and the binding of a fungal polygalacturonase by a plant polygalacturonase-inhibiting protein (Leckie et al. 1999) also point to the importance of solvent-exposed residues in the $\beta$ strand/ $\beta$-turn regions of the cognate LRR. Therefore, it would be interesting to see whether R363, predicted to a solvent-exposed residue in the $\beta$-strand region of LRR12, could contribute to the strength of Avr9 recognition observed by Wulff and associates (2009).

Only seven amino acid differences are unique to both Cf-9B and Hcr9-9A compared with Hcr9 proteins unable to trigger necrosis in N. benthamiana; namely, D200, G247, N338, D339, S356, Y372, and H391 (Fig. 7C). Of these, only D200 in LRR5, G247 in LRR7, N338 and D339 in LRR11, and H391 in LRR13 are predicted to be solvent-exposed $\beta$-strand residues. If Cf-9B and Hcr9-9A both recognize the same necrosisinducing factor in $N$. benthamiana IF, then the distribution of these residues would be consistent with the observation that residues in both LRR 10 to 12 and 13 to 15 are capable of triggering necrosis in $N$. benthamiana.

Only six amino acid differences are unique to Cf-9B: Y244, K250, E321, K364, G365, and F403 (Fig. 7C). Of these, only Y244 in LRR7, E321 in LRR10, and K364 and G365 in LRR12 are predicted to be solvent-exposed $\beta$-strand residues. If Cf-9B and Hcr9-9A recognize different necrosis-inducing factors in $N$. benthamiana IF, then the distribution of these residues would not be consistent with the observation that residues in both LRR 10 to 12 and 13 to 15 are capable of triggering necrosis in $N$. benthamiana. The lack of unique solventexposed $\beta$-strand residues in LRR 13 to 15 of Cf-9B suggests that Cf-9B and Hcr9-9A most likely recognize the same necrosis-inducing factor or that residues predicted to lie outside of the $\beta$-strand region are also involved in recognition.

\section{The relationship between Cf-9B, Hcr9-9A, and the necrosis-inducing factor from $N$. benthamiana.}

We have shown that Agrobacterium-mediated expression of Hcr9-9A or Cf-9B, but not Cf-9, induces necrosis in leaves of $N$. benthamiana and that the apoplast of $N$. benthamiana leaves contains a factor capable of inducing necrosis in leaves of transgenic tobacco (N. tabacum) plants expressing Hcr9-9A, Cf-9B, and Cf-9 but not Cf-9 alone. We postulate that either Hcr9-9A, Cf-9B, or both recognize the necrosis-inducing factor and trigger a response. Consistent with the third option, Hcr99A shows a higher degree of similarity to Cf-9B than any other Hcr9 sequenced to date $(91 \%$ amino acid identity and $94 \%$ similarity). However, Hcr9-9A is almost identical to Hcr9-4A (861/865 identical amino acids) and Hcr9-SC0A (855/865 identical amino acids). Neither has been transiently expressed in $N$. benthamiana but it seems likely that both would trigger necrosis. Hcr9-SCOA is present in $\mathrm{Cf0}$ tomato plants but $\mathrm{CfO}$ plants do not show mature plant resistance (Panter et al. 2002). This suggests that the mature plant resistance conferred by Cf9B might be a distinct attribute separable from the necrosis induction caused by $\mathrm{Cf}-9 \mathrm{~B}$ and Hcr9-9A or that $\mathrm{Cf}-9 \mathrm{~B}$ and Hcr9-9A recognize different necrosis-inducing factors or recognize the same necrosis-inducing factor in different ways.

Wulff and associates (2004) have shown that several natural Hcr 9 variants and a number of recombinant $H c r 9$ gemes generated by gene shuffling induce necrosis in various Nicotiana spp. They identified six different patterns of necrosis induction among five Nicotiana spp. (including N. tabacum and N. benthamiana) and showed that the Hcr9 proteins all induced necrosis via the same downstream signaling pathway as Cf-9. However, they were unable to correlate necrosis induction with particular combinations of polymorphic residues, suggesting that there are probably multiple ways in which activation could occur. The different patterns of necrosis induction for the various Hcr9 proteins suggested either regulation of autoactivity by different upstream signaling proteins or recognition of different plant proteins. In the case of Hcr9-9A and Cf-9B, we believe our study points to the recognition of a component of the plant apoplast related to $\mathrm{Cf}-9 \mathrm{~B}-\mathrm{mediated}$ disease resistance in tomato.

\section{MATERIALS AND METHODS}

\section{DNA manipulations and polymerase chain reaction.}

Unless otherwise stated, DNA manipulations were performed using standard methods (Sambrook et al. 1989). Polymerase chain reaction (PCR) was performed on purified plasmids or on boiled bacterial cells using REDTaq DNA polymerase and REDTaq PCR buffer (Sigma-Aldrich Co., St Louis) and an MJ Research (Watertown, MA, U.S.A.) PTC-200 thermal cycler. The orientation and authenticity of constructs was tested by diagnostic restriction analysis or by $\mathrm{PCR}$, using primers designed against sites within the $C f-9$ and $C f-9 B$ genes and in the multiple cloning sites of vectors. Junction sites in chimeric Bluescript constructs were analyzed by dye terminator sequencing, with the exception of pSLJ95622.

\section{Preparation of plasmid constructs for domain swaps and plant transformation.}

Following their isolation, the $C f-9$ and $C f-9 B$ genes were modified by removal and addition of restriction sites to facilitate domain swaps. The $C f-9$ gene in pSLJ9595, whose construction has been described previously (Benghezal et al. 2000), differs from wild-type $C f-9$ by a GAA to TCG mutation preceding the start codon that creates a ClaI site, a silent A2067G mutation in the coding sequence that removes a $B g l I I$ site, and a $\mathrm{C}$ to A mutation that removes a ClaI site in the $3^{\prime}$ untranslated region. The $C f-9 B$ gene in pSLJ10601, whose construction has also been described previously (Panter et al. 2002), differs from wild-type $C f-9 B$ by a GAA to TCG mutation preceding the start codon that creates a ClaI site, an A586G mutation that introduces a $S a c I$ site homologous to that of $C f-9$ and leads to the replacement of a glycine residue in $\mathrm{Cf}-9 \mathrm{~B}$ by a 
glutamate residue homologous to that of $\mathrm{Cf}-9$, and a silent A732G mutation that removes an EcoRI site.

The construct pSLJ9601 was made by ligating the ClaI$B a m \mathrm{HI}$ fragment containing the $C f-9$ coding and $3^{\prime}$ untranslated region from pSLJ9595 into the binary vector pSLJ10080. pSLJ10080 is a derivative of pCLD04541 (Bancroft et al. 1997) that contains the CaMV $35 \mathrm{~S}$ promoter on an EcoRIBamHI fragment derived from pSLJ4J8 (Jones et al. 1992). Other 35S gene fusions were generated using pCBJ10 (M. Benghezal and D. Jones, unpublished), which was made by ligating the ClaI-EcoRI 35S promoter fragment from pSLJ4J8 into a derivative of the binary vector pSLJ7291 (Thomas et al. 1997) with a modified polylinker that enables subsequent cloning of coding regions behind the $35 \mathrm{~S}$ promoter as $\mathrm{ClaI}$, ClaIBamHI, or ClaI-XbaI fragments. The construct pCBJ200 was made by ligating the $3.3-\mathrm{kb} C l a \mathrm{I}$ fragment containing the $C f$ $9 B$ coding and untranslated $3^{\prime}$ region from pSLJ10601 into the ClaI site of pCBJ10. pSLJ9601 and pCBJ200 were used as the $C f-9$ and $C f-9 B$ control constructs, respectively, in transient expression experiments in $N$. tabacum and $N$. benthamiana.

The construction of domain swaps between Cf-9 and Cf9B and other binary vector constructs used in this work have been described in the Supplementary Experimental Procedures.

\section{Transformation and regeneration of tomato plants.}

Binary vectors were transferred from Escherichia coli DH10 $\beta$ to A. tumefaciens LBA4404 by triparental mating, using an E. coli strain carrying the helper plasmid pRK2013 as described by Jones and associates (1992). Cells were plated on Minimal A medium (Lech and Brent 1998) containing tetracycline at $2.5 \mu \mathrm{g} / \mathrm{ml}$ and were screened for the presence of binary vectors by PCR. The Moneymaker cultivar of L esculentum, lacking known genes for resistance to $C$. fulvum, was used for Agrobacterium-mediated plant transformation, which was performed as previously described (Jones et al. 1994). Seed of transgenic plants were screened for kanamycin resistance on Murashige-Skoog (MS) media containing kanamycin sulfate (100 $\mu \mathrm{g} / \mathrm{ml})$, which provided selection for plants expressing the nptII gene contained within the binary vectors (Jones et al. 1992).

\section{Inoculation of tomato plants with $C$. fulvum.}

C. fulvum cultures were prepared as described by Hammond-Kosack and Jones (1993) and were used to make spore suspensions containing Silwet L-77 at $0.5 \mathrm{ml} /$ liter (Osi Specialties, Sistersville, WV, U.S.A.) for plant inoculations. The apical buds of plants used for inoculation at the fruiting stage were trimmed to leave one to three leaves above the first flowering truss. Plants at the two- to four-true-leaf or early fruiting stages of development were treated with paclobutrazol and inoculated as previously described (Balint-Kurti et al. 1994). After inoculation, mature plants were placed on a saturated capillary mat within a sealed $90-$ by-90-by-80-cm plasticcovered frame designed to maximize humidity. A similar 90by-90-by-50-cm frame was used for seedling inoculations. The plastic covering these frames was perforated after 3 days to reduce the humidity slightly and to allow gas exchange for the remainder of the incubation period. Plants were scored for macroscopic signs of infection 21 to 24 days after inoculation and leaf samples were stained with lactophenol trypan blue (Hammond-Kosack and Jones 1993).

\section{Transient gene expression by agroinfiltration.}

Binary vectors based on pCBJ10 were transferred from $E$. coli to the $A$. tumefaciens strain GV3101 by triparental mating, as already described. Mating mixtures were plated on M9 medium containing tetracycline at $2.5 \mu \mathrm{g} / \mathrm{ml}$ and rifampicin at 50 $\mu \mathrm{g} / \mathrm{ml}$, and colonies were screened for the presence of the binary vector by PCR. Binary vectors based on pGREENII were co-electroporated along with pSOUP into electrocompetent GV3101. Electroporated cells were plated on Luria-Bertani (LB) medium containing rifampicin $(50 \mu \mathrm{g} / \mathrm{ml})$, gentamicin $(50 \mu \mathrm{g} / \mathrm{ml})$, tetracycline $(5 \mu \mathrm{g} / \mathrm{ml})$, and kanamycin $(50 \mu \mathrm{g} / \mathrm{ml})$ and colonies were screened for the presence of the binary vector by PCR. Prior to agroinfiltration, A. tumefaciens cell lines were grown for $16 \mathrm{~h}$ at $28^{\circ} \mathrm{C}$ with shaking in $2 \mathrm{ml}$ of $\mathrm{LB}$ medium with rifampicin at $50 \mu \mathrm{g} / \mathrm{ml}$ and tetracycline at 0.5 $\mu \mathrm{g} / \mathrm{ml}$. A $1-\mathrm{ml}$ aliquot of each culture was used to inoculate 40 $\mathrm{ml}$ of LB media containing Difco Bacto tryptone at $10 \mathrm{~g} / \mathrm{liter}$; Difco Bacto yeast extract at $1 \mathrm{~g} / \mathrm{liter}$; $\mathrm{NaCl}$ at $5 \mathrm{~g} / \mathrm{liter}$; D-glucose at $1 \mathrm{~g} /$ liter; $10 \mathrm{mM}$ MES, pH 5.6; $20 \mu \mathrm{M}$ acetosyringone; and tetracycline at $0.5 \mu \mathrm{g} / \mathrm{ml}$. Cultures were grown at $28^{\circ} \mathrm{C}$ for $18 \mathrm{~h}$ and cells were washed in $10 \mathrm{ml}$ of MS basal salts with $500 \mu \mathrm{M}$ MES, pH 5.6; $2 \%$ sucrose; $0.1 \%$ glucose; and $10 \mu \mathrm{M}$ acetosyringone before being resuspended in the same buffer to an absorbance value at $600 \mathrm{~nm}$ of $1 \pm 0.05$. Cell suspensions were incubated at $22^{\circ} \mathrm{C}$ for $3 \mathrm{~h}$ before being infiltrated into leaves of mature $N$. tabacum or $N$. benthamiana plants as described previously (Thomas et al. 1999; Van der Hoorn et al. 1999).

\section{Extraction of intercellular fluids \\ from tobacco or infected tomato leaves.}

IF were extracted from tomato and tobacco leaves essentially as described by de Wit and Spikman (1982). Leaves of Moneymaker tomato plants that had been infected with $C$. fulvum race 5,9 were harvested 20 to 24 days after inoculation. This IF was purified of any remaining plant debris and fungal spores using a $0.2-\mu \mathrm{m}$ pore-size cartridge filter and stored at $-20^{\circ} \mathrm{C}$. The same procedure was used to extract IF from uninfected Moneymaker tomato plants and from leaves of flowering $N$. benthamiana plants. IF preparations were centrifuged briefly at maximum speed in a microcentrifuge just before use in order to remove suspended solids and were injected into leaves as described by Brading and associates (2000). Where indicated, the IF from $N$. benthamiana was concentrated by freeze drying and resuspending in $20 \mathrm{mM}$ phosphate buffer ( $\mathrm{pH} 5.5)$ containing $100 \mathrm{mM} \mathrm{NaCl}$ prior to injection.

\section{ACKNOWLEDGMENTS}

We thank J. Wilson for plant photography, S. Lyons and S. Dempsey for horticultural assistance, J. Neale and his workshop team for producing inoculation frames, and A. Hardham for assistance with light microscopy. S. Panter was supported by an Australian Postgraduate Award. A. Charabarti was supported by an Australian National University Ph.D. scholarship.

\section{LITERATURE CITED}

Balint-Kurti, P. J., Dixon, M. S., Jones, D. A., Norcott, K. A., and Jones, J. D. G. 1994. RFLP linkage analysis of the $C f-4$ and $C f-9$ genes for resistance to Cladosporium fulvum in tomato. Theor. Appl. Genet. 88:691700.

Bancroft, I., Love, K., Bent, E., Sherson, S., Lister, C., Cobett, C., Goodman, H. M., and Dean, C. 1997. A strategy involving the use of high redundancy YAC subclone facilitates the contiguous representation in cosmid and BAC clones of $1.7 \mathrm{Mb}$ of the genome of the plant Arabidopsis thaliana. Weeds World 4:1-9.

Belfanti, E., Silfverberg-Dilworth, E., Tartarini, S., Patocchi, A., Barbieri, M., Zhu, J., Vinatzer, B. A., Gianfranceschi, L., Gessler, C., and Sansavini, S. 2004 The HcrVf2 gene from a wild apple confers scab resistance to a transgenic cultivated variety. Proc. Natl. Acad. Sci. U.S.A. 101:886-890.

Benghezal, M., Wasteneys, G. O., and Jones, D. A. 2000. The C-terminal dilysine motif confers endoplasmic reticulum localisation to type I membrane proteins in plants. Plant Cell 12:1179-1201.

Brading, P. A., Hammond-Kosack, K. E., Parr, A., and Jones, J. D. G. 2000. Salicylic acid is not required for $C f$-2- and $C f$-9-dependent resis- 
tance of tomato to Cladosporium fulvum. Plant J. 23:305-318.

de Wit, P. J. G. M., and Spikman, G. 1982. Evidence for the occurrence of race and cultivar-specific elicitors of necrosis in intercellular fluids of compatible interactions of Cladosporium fulvum and tomato. Physiol. Plant Pathol. 21:1-11.

Dixon, M. S., Jones, D. A., Keddie, J. S., Thomas, C. M., Harrison, K., and Jones, J. D. G. 1996. The tomato $C f$-2 disease resistance locus comprises two functional genes encoding leucine-rich repeats. Cell 84:451459.

Dixon, M. S., Hatzixanthis, K., Jones, D. A., Harrison, K., and Jones, J. D. G. 1998. The tomato $C f-5$ disease resistance gene and six homologs show pronounced allelic variation in leucine-rich repeat copy number. Plant Cell 10:1915-1925.

Dunning, F. M., Sun, W., Jansen, K. L., Helft, L., and Bent, A. F. 2007. Identification and mutational analysis of Arabidopsis FLS2 leucine-rich repeat domain residues that contribute to flagellin perception. Plant Cell 19:3297-3313.

Hammond-Kosack, K. E., and Jones, J. D. G. 1993. Incomplete dominance of tomato $C f$ genes for resistance to Cladosporium fulvum. Mol. PlantMicrobe Interact. 7:58-70.

Hammond-Kosack, K. E., Tang, S., Harrison, K., and Jones, J. D. G. 1998 The tomato $C f-9$ disease resistance gene functions in tobacco and potato to confer responsiveness to the fungal avirulence gene product Avr9. Plant Cell 10:1251-1266.

Jones, D. A., Thomas, C. M., Hammond-Kosack, K. E., Balint-Kurti, P. J., and Jones, J. D. G. 1994. Isolation of the tomato $C f-9$ gene for resistance to Cladosporium fulvum by transposon tagging. Science 266:789793

Jones, J. D. G., Shlumukov, L., Carland, F., English, J., Scofield, S. R., Bishop, G. J., and Harrison, K. 1992. Effective vectors for transformation, expression of heterologous genes, and assaying transposon excision in transgenic plants. Transgenic Res. 1:285-297.

Kawchuk, L. M., Hachey, J., Lynch, D. R., Kulcsar, F., van Rooijen, G., Waterer, D. R., Robertson, A., Kokko, E., Byers, R., Howard, R. J., Fischer, R., and Prüfer, D. 2001 Tomato Ve disease resistance genes encode cell surface-like receptors. Proc. Natl. Acad. Sci. U.S.A. 98:65116515 .

Kooman-Gersmann, M., Honee, G., Bonnema, G., and de Wit, P. G. J. M 1996. A high-affinity binding site for the AVR9 peptide elicitor of Cladosporium fulvum is present on plasma membranes of tomato and other Solanaceous plants. Plant Cell 8:929-938.

Lech, K., and Brent, R. 1998. Escherichia coli, plasmids and bacteriophages. Pages S27/1.1.2 in: Current Protocols in Molecular Biology, Vol. 1. F. M. Ausubel, R. Brent, R. E. Kingston, D. D. Moore, J. G. Seidman, J. A. Smith, K. Struhl, L. M. Albright, D. M. Coen, A. Varki, and V. B. Chanda, eds. John Wiley and Sons, New York.

Leckie, F., Mattei, B., Capodicasa, C., Hemmings, A., Nuss, L.,. Aracri, B, De Lorenzo, G., and Cervone, F. 1999. The specificity of polygalacturonase-inhibiting protein (PGIP): A single amino acid substitution in the solvent-exposed $\beta$-strand/ $\beta$-turn region of the leucine-rich repeats (LRRs) confers a new recognition capability. EMBO (Eur. Mol. Biol. Organ.) J. 18:2352-2363.

Luderer, R., Rivas, S., Nurnberger, T., Mattei, B., Van den Hooven, H. W. Van der Hoorn, R. A. L., Romeis, T., Wehrfritz, J.-M., Blume, B., Nennstiel, D., Zuidema, D., Vervoort, J., De Lorenzo G., Jones, J. D. G., de Wit, P. J. G. M., and Joosten, M. H. A. J. 2001. No evidence for binding between resistance gene product $\mathrm{Cf}-9$ of tomato and avirulence gene product AVR9 of Cladosporium fulvum. Mol. Plant-Microbe Interact. 14:867-876.

Marmeisse, R., Van den Ackerveken, G. F. J. M., Goosen, T., de Wit, P. J. G. M., and Van den Broeck, H. W. J. 1993. Disruption of the avirulence gene Avr9 in 2 races of the tomato pathogen Cladosporium fulvum causes virulence on tomato genotypes with the complementary resistance gene $C f-9$. Mol. Plant-Microbe Interact. 6:412-417.

Panter, S. N., Hammond-Kosack, K. E., Harrison, K., Jones, J. D. G., and Jones, D. A. 2002 Developmental control of promoter activity is not re- sponsible for mature onset of $C f-9 B$-mediated resistance to leaf mold in tomato. Mol. Plant-Microbe Interact. 15:1099-1107.

Parniske, M., Hammond-Kosack, K. E., Golstein, C., Thomas, C. M., Jones, D. A., Harrison, K., Wulff, B. B. H., and Jones, J. D. G. 1997. Novel disease resistance specificities result from sequence exchange between tandemly repeated genes at the $\mathrm{Cf}-4 / 9$ locus of tomato. Cell 91:821-832.

Parniske, M., Wulff, B. H. H., Bonnema, G., Thomas, C. M., Jones, D. A and Jones, J. D. G. 1999. Homologues of the Cf-9 disease resistance gene (Hcr9s) are present at multiple loci on the short arm of tomato chromosome 1. 12:93-102.

Piedras, P., Rivas, S., Dröge, S., Hillmer, S., and Jones, J. D. G. 2000. Functional, c-myc-tagged $C f-9$ resistance gene products are plasmamembrane localised and glycosylated. Plant J. 21:529-536.

Sambrook, J., Fritsch, E. F., and Maniatis, T. 1989. Molecular Cloning: A Laboratory Manual. Cold Spring Harbor Press, Cold Spring Harbor, NY, U.S.A

Song, W. Y., Wang, G. L., Chen, L. L., Kim, H. S., Pi, L. Y., Holsten, T. Gardner, J., Wang, B., Zhai, W. X., Zhu, L. H., Fauquet, C., and Ronald, P. 1995. A receptor kinase-like protein encoded by the rice disease resistance gene, $\mathrm{Xa21}$. Science 270:1804-1806.

Takken, F. L. W., Thomas, C. M., Joosten, M. H. A. J., Golstein, C., Westerink, N., Hille, J., Nijkamp, H. J. J., de Wit, P. J. G. M., and Jones, J. D. G. 1999 A second gene at the tomato $C f$-4 locus confers resistance to Cladosporium fulvum through recognition of a novel avirulence determinant. Plant J. 20:279-288.

Thomas, C. M., Jones, D. A., Parniske, M., Harrison, K., Balint-Kurti, P. J., Hatzixanthis, K., and Jones, J. D. G. 1997. Characterisation of the tomato $C f-4$ gene for resistance to Cladosporium fulvum identifies sequences that determine recognitional specificity in $C f-4$ and $C f-9$. Plant Cell 9:2209-2224.

Thomas, C. M., Tang, S., Hammond-Kosack, K., and Jones, J. D. G. 1999. Comparison of the hypersensitive response induced by the tomato $C f-4$ and $C f-9$ genes in Nicotiana spp. Mol. Plant-Microbe Interact. 13:465469.

Van der Hoorn, R. A. L., Laurent, F., Roth, R., and de Wit, P. J. G. M. 1999. Agroinfiltration is a versatile tool that facilitates comparative analyses of $A v r 9 / C f$-9-Induced and Avr4/Cf-4-induced necrosis. Mol. Plant-Microbe Interact. 13:439-446.

Van der Hoorn, R. A. L., Kruijt, M., Roth, R., Brandwagt, B. F., Joosten, M. H. A. J., and de Wit, P. J. G. M. 2001a. Intragenic recombination generated two distinct $C f$ genes that mediate AVR9 recognition in the natural population of Lycopersicon pimpinellifolium. Proc. Natl. Acad Sci. U.S.A. 98:10493-10498

Van der Hoorn, R. A. L., Roth, R., and de Wit, P. J. G. M. 2001b. Identification of distinct specificity determinants in resistance protein $\mathrm{Cf}-4$ allows construction of a Cf-9 mutant that confers recognition of avirulence protein AVR4. Plant Cell 13:273-285.

Van der Hoorn, R. A. L., Wulff, B. B. H., Rivas, S., Durrant, M. C., van der Ploeg, A., de Wit, P. J. G. M., and Jones, J. D. G. 2005 Structurefunction analysis of Cf-9, a receptor-like protein with extracytoplasmic leucine-rich repeats. Plant Cell 17:1000-1015.

Wulff, B. B. H., Thomas, C. M., Smoker, M., Grant, M., and Jones, J. D. G. 2001. Domain swapping and gene shuffling identify sequences required for induction of an Avr-dependent hypersensitive response by the tomato Cf-4 and Cf-9 proteins. Plant Cell 13:255-272.

Wulff, B. B. H., Kruijt, M., Collins, P. L., Thomas, C. M., Ludwig, A. A., de Wit P. J. G. M., and Jones, J. D. G. 2004. Gene shuffling-generated and natural variants of the tomato resistance gene Cf- 9 exhibit different auto-necrosis-inducing activities in Nicotiana species. Plant J. 40:942956.

Wulff, B. B. H., Heese-Peck, A., Buhot, L., Jones, D. A., Jones, and J. D. G. 2009. The specificity-determining amino acids of the tomato Cf-9 disease resistance protein are at hypervariable putative solvent-exposed positions in the central leucine-rich repeats. Mol. Plant-Microbe Interact. 22:1203-1213 